\title{
EXPLICIT CONSTRUCTION OF THE MODIFIED SPHERICAL HARMONIC SERIES FOR THE GRAVITY GRADIENTS AND ANALYSIS OF THEIR CHARACTERISTICS
}

\author{
M. S. Petrovskaya, A. N. Vershkov \\ Central (Pulkovo) Astronomical Observatory of the Russian Academy of Sciences \\ Pulkovskoe Shosse 65/1, St. Petersburg, 196140, Russia \\ e-mail: petrovsk@gao.spb.ru \\ Tel.: +7-812-7234422, Fax: +7-812-3143360
}

\begin{abstract}
The present paper complements the research carried out in PV2008 (Petrovskaya and Vershkov, 2008), concerning the expansion of the gravity gradients in the local northoriented reference frame in orthogonal series of modified spherical harmonics. In PV2008 procedures are developed for recovering the orthogonal bases of these series. Then an idea is briefly described how the spectral relations can be obtained between the gravity gradients and the geopotential. However no explicit procedures are demonstrated for their derivation. In the present paper successive transformations are described for each derivative which convert the initial non-orthogonal expansion into the orthogonal series. The resulting spectral relations are applied for evaluating the harmonic coefficients of these series at different altitudes, on the basis of the geopotential model EGM2008. The corresponding degree variances are estimated. The new simple expressions for the gravity gradients are convenient for various applications. In the present paper they are implemented for constructing digital colored maps for Fennoscandia region which attracts much attention of geophysicists. These maps visually demonstrate an anomalous behavior of the gravity gradients in this area.
\end{abstract}

Keywords. Gravity gradients · Modified spherical harmonic expansions · Spectral coefficients - Degree variances - Digital maps for the gravity gradients in Fennoscandia region

\section{INTRODUCTION}

The Earth's disturbing potential $T=V-U$ is considered where $V$ represents the true gravitational potential and $U$ is the normal one.

The expressions for the second-order derivatives of the potential $T$ (gravity gradients) are studied in the local north-oriented reference frame $\{x, y, z\}$, where $z$ has the geocentric radial direction, $x$ points to the north, $y$ is directed to the west.

The conventional expressions for these derivatives depend not only on the associated Legendre functions $\bar{P}_{n,|m|}(\cos \theta)$ of the colatitude $\theta$ but also on their first and second order derivatives. Besides, the factors at the Legendre functions and at their derivatives tend to infinity when approaching the poles. In the paper PV2006 (Petrovskaya and Vershkov, 2006) alternative expressions for the gravity gradients are constructed which do not have the above 
properties and can be applied for any satellite orbit, which can pass the polar regions. These expressions depend directly on the coefficients of the geopotential but are not orthogonal.

In the paper PV2008 (Petrovskaya and Vershkov, 2008) a number of researchers are mentioned who propose various orthogonal basis for expanding the second order derivatives of the geopotential. In PV2008 more simple orthogonal bases are recovered for these derivatives. They represent two sets of modified spherical harmonics which only slightly differ from the conventional spherical functions. One orthogonal basis corresponds to the tangential derivatives $T_{x x}, T_{y y}, T_{x y}$ and the other to $T_{x z}$ and $T_{y z}$. The dependence of the modified harmonics on the longitude $\lambda$ is the same as in the conventional spherical functions. The only difference between the standard spherical harmonics and the modified harmonics is that the index $|m|$ in the functions $\bar{P}_{n,|m|}(\cos \theta)$ is one or two units less or larger than in $\sin m \lambda$ and $\cos m \lambda$. The maximal degree of harmonics in the orthogonal series is the same as in the truncated conventional expansions and in the expansions constructed in PV2006.

In PV2008 an approach is briefly described to constructing the orthogonal series. This approach, unified for all the potential derivatives, is demonstrated on an example of $T_{y y}$. Several implicit and two explicit intermediate equations are presented for this derivative. For the other derivatives, $T_{x x}, T_{x y}, T_{x z}$ and $T_{x x}$, only a brief comment is given. For all the derivatives the final spectral relations are provided, which connect the coefficients of the derivatives and the geopotential.

In the present paper explicit transformations for all the derivatives are described which result in the spectral relations, the same as in PV2008. It is described how on the basis of these relations two mutually inverse problems can be solved: synthesizing the spectra of the derivatives from a known geopotential model and evaluating the geopotential coefficients from a known spectrum of each derivative.

The simple orthogonal series are very convenient for recovering various characteristics of the gravity gradients. The coefficients of these series and the corresponding degree variances are evaluated on the basis of the geopotential model EGM2008 (Pavlis et al., 2008). Colored digital maps demonstrate the anomalous behavior of the gravity gradients in Fennoscandia region, which is of much interest for geophysicists.

\section{NON-ORTHOGONAL AND ORTHOGONAL EXPANSIONS FOR THE GRAVITY GRADIENTS}

A truncated spherical harmonic series for the disturbing potential $T$ is considered

$$
T=\frac{\mu}{a} \sum_{n=2}^{N} \sum_{|m|=0}^{n}\left(\frac{a}{r}\right)^{n+1} \bar{C}_{n, m} \bar{Y}_{n, m}(\theta, \lambda)
$$

where $\bar{Y}_{n, m}$ are the surface spherical harmonics

$$
\bar{Y}_{n, m}(\theta, \lambda)=\bar{P}_{n,|m|}(\cos \theta) Q_{m}(\lambda), \quad Q_{m}(\lambda)=\left\{\begin{array}{cc}
\cos m \lambda, & m \geq 0 \\
\sin |m| \lambda, & m<0 .
\end{array}\right.
$$

In the above equations $r, \theta, \lambda$ are the spherical coordinates. The quantity $\mu=G M$ is the gravitational constant multiplied by the Earth's mass, $a$ is the semi-major axis of the reference ellipsoid, $\bar{C}_{n, m}$ and $\bar{P}_{n,|m|}(\cos \theta)$ represent the fully normalized potential coefficients 
and the associated Legendre functions, respectively. In (2) and the subsequent equations the summation with respect to $|m|$ includes both positive and negative values of $m$.

From (1) the expansion for the second order radial derivative follows

$$
T_{z z}=\frac{\mu}{a^{3}} \sum_{n=2}^{N} \sum_{|m|=0}^{n}(n+1)(n+2)\left(\frac{a}{r}\right)^{n+3} \bar{C}_{n, m} \bar{Y}_{n, m}(\theta, \lambda) .
$$

In PV2006 simple, non-singular expressions for the other second order derivatives of the disturbing potential are derived which are presented here as Fourier series

$$
\begin{aligned}
T_{x x}= & \frac{\mu}{a^{3}} \sum_{|m|=0}^{N} \sum_{n=\max \{2,|m|\}}^{N}\left(\frac{a}{r}\right)^{n+3} \bar{C}_{n, m} Q_{m}(\lambda)\left\{a_{n, m} \bar{P}_{n,|m|-2}(\cos \theta)\right. \\
& \left.+\left[b_{n, m}-(n+1)(n+2)\right] \bar{P}_{n,|m|}(\cos \theta)+c_{n, m} \bar{P}_{n,|m|+2}(\cos \theta)\right\}, \\
T_{y y}= & -\frac{\mu}{a^{3}} \sum_{|m|=0}^{N} \sum_{n=\max \{2,|m|\}}^{N}\left(\frac{a}{r}\right)^{n+3} \bar{C}_{n, m} Q_{m}(\lambda)\left[a_{n, m} \bar{P}_{n,|m|-2}(\cos \theta)\right. \\
+ & \left.b_{n, m} \bar{P}_{n,|m|}(\cos \theta)+c_{n, m} \bar{P}_{n,|m|+2}(\cos \theta)\right], \\
T_{x y}= & \frac{\mu}{a^{3}} \sum_{|m|=1}^{N} \sum_{n=\max \{2,|m|\}}^{N}\left(\frac{a}{r}\right)^{n+3} \bar{C}_{n, m} Q_{-m}(\lambda)\left[d_{n, m} \bar{P}_{n-1,|m|-2}(\cos \theta)\right. \\
& \left.+g_{n, m} \bar{P}_{n-1,|m|}(\cos \theta)+h_{n, m} \bar{P}_{n-1,|m|+2}(\cos \theta)\right], \\
T_{x z}= & \frac{\mu}{a^{3}} \sum_{|m|=0}^{N} \sum_{n=\max \{2,|m|\}}^{N}\left(\frac{a}{r}\right)^{n+3} \bar{C}_{n, m} Q_{m}(\lambda) \\
& \times\left[\beta_{n, m} \bar{P}_{n,|m|-1}(\cos \theta)+\gamma_{n, m} \bar{P}_{n,|m|+1}(\cos \theta)\right], \\
T_{y z}= & \frac{\mu}{a^{3}} \sum_{|m|=1}^{N} \sum_{n=\max \{2,|m|\}}^{N}\left(\frac{a}{r}\right)^{n+3} \bar{C}_{n, m} Q_{-m}(\lambda) \\
& \times\left[\mu_{n, m} \bar{P}_{n-1,|m|-1}(\cos \theta)+v_{n, m} \bar{P}_{n-1,|m|+1}(\cos \theta)\right] .
\end{aligned}
$$

The numerical constants in (4) and in the subsequent equations are given in the Appendix.

The radial derivative (3) is rewritten in a more compact form

$$
T_{z z}=\sum_{|m|=0}^{N} \sum_{n=\max \{2,|m|\}}^{N} \bar{R}_{n, m} \bar{Y}_{n, m}(\theta, \lambda),
$$

where the quantities

$$
\bar{R}_{n, m}=\frac{\mu}{a^{3}}(n+1)(n+2)\left(\frac{a}{r}\right)^{n+3} \bar{C}_{n, m}
$$

are introduced, instead of the coefficients $\bar{C}_{n, m}$, in order to simplify the subsequent transformations and computations.

The orthogonal expansions considered in PV2008 and in the present paper have the form 


$$
\begin{aligned}
& T_{x x}=\sum_{|m|=0}^{N} \sum_{n=|m|}^{N+2} \bar{H}_{n, m} \bar{Z}_{n, m}(\theta, \lambda), \\
& T_{y y}=\sum_{|m|=0}^{N} \sum_{n=|m|}^{N+2} \bar{G}_{n, m} \bar{Z}_{n, m}(\theta, \lambda), \\
& T_{x y}=\sum_{|m|=1}^{N} \sum_{n=|m|}^{N+1} \bar{M}_{n, m} \bar{Z}_{n, m}(\theta, \lambda), \\
& T_{x z}=\sum_{|m|=0}^{N} \sum_{n=|m|}^{N+1} \bar{B}_{n, m} \bar{X}_{n, m}(\theta, \lambda), \\
& T_{y z}=\sum_{|m|=1}^{N} \sum_{n=|m|}^{N} \bar{A}_{n, m} \bar{X}_{n, m}(\theta, \lambda) .
\end{aligned}
$$

The coefficients of these series depend on the quantities $\bar{R}_{n, m}$ defined in Eq. (6), as will be shown in Section 3.

For the tangential derivatives, the modified spherical harmonics have the form

$$
\left.\begin{array}{l}
\bar{Z}_{n, m}(\theta, \lambda)=\bar{P}_{n,|m|}(\cos \theta) Q_{m}(\lambda),|m|=0,1, \\
\bar{Z}_{n, m}(\theta, \lambda)=\bar{P}_{n-2,|m|-2}(\cos \theta) Q_{m}(\lambda),|m|=2,3, \ldots, N .
\end{array}\right\}
$$

For the derivatives $T_{x z}$ and $T_{y z}$, the harmonics are

$$
\left.\begin{array}{l}
\bar{X}_{n, 0}(\theta, \lambda)=\bar{P}_{n, 1}(\cos \theta), \\
\bar{X}_{n, m}(\theta, \lambda)=\bar{P}_{n-1,|m|-1}(\cos \theta) Q_{m}(\lambda),|m|=1,2, \ldots, N .
\end{array}\right\}
$$

The quantities in (7)-(13) are fully normalized by the conventional rules.

It can be easily established that the harmonics $\bar{Z}_{n, m}(\theta, \lambda)$ and $\bar{X}_{n, m}(\theta, \lambda)$ are orthonormal, similarly to the conventional spherical harmonics $\bar{Y}_{n, m}(\theta, \lambda)$. For example, the functions $\bar{Z}_{n, m}(\theta, \lambda)$ from (12) for $|m|=0,1$ represent the conventional spherical harmonics $\bar{Y}_{n, m}(\theta, \lambda)$. For $|m|=2,3, \ldots, N$ these functions are also orthonormal:

$$
\begin{aligned}
I_{n, m, l, k} & =\frac{1}{4 \pi} \int_{\sigma} \bar{Z}_{l, k}(\theta, \lambda) \bar{Z}_{n, m}(\theta, \lambda) \mathrm{d} \sigma \\
& =\frac{1}{4 \pi} \int_{0}^{2 \pi} Q_{k}(\lambda) Q_{m}(\lambda) \mathrm{d} \lambda \int_{-1}^{1} \bar{P}_{l,|k|-2}(x) \bar{P}_{n,|m|-2}(x) \mathrm{d} x=\delta_{k, m} \delta_{l, n} .
\end{aligned}
$$

where $\sigma$ is the unite sphere, $x=\cos \theta$ and $\delta_{p, q}$ is the Kronecker symbol.

A similar equation exists for the basic functions $\bar{X}_{n, 0}(\theta, \lambda)$. For $|m|=1,2, \ldots, N$ we have: 


$$
\begin{aligned}
J_{n, m, l, k} & =\frac{1}{4 \pi} \int_{\sigma} \bar{X}_{l, k}(\theta, \lambda) \bar{X}_{n, m}(\theta, \lambda) \mathrm{d} \sigma \\
& =\frac{1}{4 \pi} \int_{0}^{2 \pi} Q_{k}(\lambda) Q_{m}(\lambda) \mathrm{d} \lambda \int_{-1}^{1} \bar{P}_{l,|k|-1}(x) \bar{P}_{n,|m|-1}(x) \mathrm{d} x=\delta_{k, m} \delta_{l, n} .
\end{aligned}
$$

Since the modified spherical harmonics are orthonormal, then the harmonic coefficients in (7)-(11) can be determined by integral formulas. In particular,

$$
\bar{H}_{n, m}=\frac{1}{4 \pi} \int_{\sigma} T_{x x}(\theta, \lambda) \bar{Z}_{n, m}(\theta, \lambda) \mathrm{d} \sigma .
$$

\section{RELATIONS BETWEEN THE SPECTRA OF THE DERIVATIVES AND OF THE GEOPOTENTIAL}

In PV2008 a unified approach is briefly described for constructing the orthogonal series. It is proposed to apply the final expressions in PV2006, given now in (4), for deriving from them the spectral relations between the coefficients of the orthogonal series and the quantities $\bar{R}_{n, m}$ which are functions of $r$ and the potential coefficients $\bar{C}_{n, m}$, according to equation (6). As an example, transformations (26)-(30) in PV2008, having an implicit form, are provided for the derivative $T_{y y}$ in Section 4.1 and two explicit intermediate expressions, (35) and (41), are given in Section 4.2 of PV2008. For the other derivatives, $T_{x x}, T_{x y}, T_{x z}$ and $T_{y z}$, no transformations are demonstrated which lead to the final spectral relations. In PV2008 these relations are given for $T_{y y}$ in Section 4.2 and for the other derivatives in the Appendix.

In this Section the successive procedures of constructing the orthogonal series are described for all the derivatives. We proceed now not from the mentioned above final expressions in PV2006 but from an intermediate equation for each derivative from this paper. It allows to essentially reduce the number of the transformations as compared to the unified approach.

\subsection{DERIVATIVE $T_{y y}$}

We begin from the derivative $T_{y y}$ since its expression in (4) is more simple than for $T_{x x}$.

An intermediate equation (18) from PV2006 is applied, as an initial one. We rewrite it in the form

$$
\begin{aligned}
\sin ^{2} \theta T_{y y}= & -\frac{\mu}{a^{3}} \sum_{|m|=0}^{N} \sum_{n=\max \{2,|m|\}}^{N}\left(\frac{a}{r}\right)^{n+3} C_{n, m} \\
& \left.\times\left[|m|(|m|-1) P_{n,|m|}(\cos \theta)+\sin \theta P_{n+1,|m|+1}(\cos \theta)\right]\right] Q_{m}(\lambda) .
\end{aligned}
$$

The transformation (Z.1.39) from (Ilk, 1983)

$$
\sin \theta P_{n,|m|}=\frac{(n+|m|)(n+|m|-1)}{(2 n+1)} P_{n-1,|m|-1}-\frac{(n-|m|+1)(n-|m|+2)}{(2 n+1)} P_{n+1,|m|-1}
$$

is applied to the second term in the square brackets in (17). It gives 


$$
\begin{aligned}
\sin ^{2} \theta T_{y y}= & -\frac{\mu}{a^{3}} \sum_{|m|=0}^{N} \sum_{n=\max \{2,|m|\}}^{N}\left(\frac{a}{r}\right)^{n+3} C_{n, m}\left[\left(n+2 m^{2}+1\right) \frac{(n+2)}{(2 n+3)} P_{n,|m|}(\cos \theta)\right. \\
& \left.-\frac{(n-|m|+1)(n-|m|+2)}{(2 n+3)} P_{n+2,|m|}(\cos \theta)\right] Q_{m}(\lambda) .
\end{aligned}
$$

The following transformations of equation (19) are performed. The expression in the right-hand side is presented as a sum of two double series. In the second one the index of summation $n$ is 'shifted' in such a way that the general terms in both series depend on the same Legendre function $P_{n,|m|}(\cos \theta)$. Then a transfer is performed to the fully normalized quantities and the geopotential coefficients $\bar{C}_{n, m}$ are expressed in terms of the quantities $\bar{R}_{n, m}$ defined in (6).

In the result, the following spherical harmonic series is derived from (19)

$$
\sin ^{2} \theta T_{y y}=\sum_{|m|=0}^{N} \sum_{n=\max \{2,|m|\}}^{N+2} \bar{D}_{n, m} \bar{Y}_{n, m}(\theta, \lambda) .
$$

Here

$$
\begin{aligned}
& \sum_{|m|=0}^{N} \sum_{n=\max \{2,|m|\}}^{N+2} \bar{D}_{n, m} \bar{Y}_{n, m}(\theta, \lambda)= \\
& =\sum_{|m|=0}^{N} \sum_{n=\max \{4,|m|+2\}}^{N+2} \bar{g}_{n, m} \bar{R}_{n-2, m} \bar{Y}_{n, m}(\theta, \lambda)-\sum_{|m|=0}^{N} \sum_{n=\max \{2,|m|\}}^{N} \bar{h}_{n, m} \bar{R}_{n, m} \bar{Y}_{n, m}(\theta, \lambda) .
\end{aligned}
$$

The same right-hand side in (20) will be expressed in terms of the coefficients $\bar{G}_{n, m}$ of the orthogonal series in (8).

We present the expression for the derivative $T_{y y}$ from (4) in the form

$$
T_{y y}=T_{y y}^{(1)}+T_{y y}^{(2)}
$$

where $T_{y y}^{(1)}$ corresponds to $|m|=0,1$ and $T_{y y}^{(2)}$ to $|m|=2,3, \ldots, N$.

From equation (96) in Appendix follows that $a_{n, m}=0$ for $|m|=0,1$. Then from the second equation in (4) we have

$$
T_{y y}^{(1)}=-\frac{\mu}{a^{3}} \sum_{|m|=0}^{1} \sum_{n=2}^{N}\left(\frac{a}{r}\right)^{n+3} \bar{C}_{n, m}\left[b_{n, m} \bar{P}_{n,|m|}(\cos \theta)+c_{n, m} \bar{P}_{n,|m|+2}(\cos \theta)\right] Q_{m}(\lambda) .
$$

The second component in (22), according to the second equation from (4), is

$$
\begin{aligned}
T_{y y}^{(2)}= & -\frac{\mu}{a^{3}} \sum_{|m|=2}^{N} \sum_{n=|m|}^{N}\left(\frac{a}{r}\right)^{n+3} \bar{C}_{n, m}\left[a_{n, m} \bar{P}_{n,|m|-2}(\cos \theta)\right. \\
& \left.+b_{n, m} \bar{P}_{n,|m|}(\cos \theta)+c_{n, m} \bar{P}_{n,|m|+2}(\cos \theta)\right] Q_{m}(\lambda) .
\end{aligned}
$$

The function $T_{y y}^{(1)}$ in (23) is expanded in the series of the conventional spherical harmonics, defined in (8) and (12), 


$$
T_{y y}^{(1)}=\sum_{|m|=0}^{1} \sum_{n=|m|}^{N+2} \bar{G}_{n, m} \bar{P}_{n, m}(\cos \theta) Q_{m}(\lambda) .
$$

After transferring in (25) to the non-normalized quantities, both parts of this equation are multiplied by $\sin ^{2} \theta$. It gives

$$
\sin ^{2} \theta T_{y y}^{(1)}=\sum_{|m|=0}^{1} \sum_{n=|m|}^{N+2} G_{n, m} \sin ^{2} \theta P_{n, m}(\cos \theta) Q_{m}(\lambda) .
$$

The right-hand side of (26) is transformed to a conventional spherical harmonic series.

For $n=0,1$ we have

$$
\left.\begin{array}{rl}
\sin ^{2} \theta & =\frac{2}{3}-\frac{2}{3} P_{2,0} \\
\sin ^{2} \theta P_{1,0} & =\frac{2}{5} P_{1,0}-\frac{2}{5} P_{3,0} \\
\sin ^{2} \theta P_{1,1} & =\frac{4}{5} P_{1,1}-\frac{2}{15} P_{3, \cdot}
\end{array}\right\}
$$

For $n \geq 2$ we apply equation (15) from Claessens (2005)

$$
\sin ^{2} \theta P_{n,|m|}=\tilde{a}_{n . m} P_{n+2,|m|}+\tilde{b}_{n . m} P_{n,|m|}+\tilde{c}_{n, m} P_{n-2,|m|} .
$$

Expressions (27) and (28) are substituted in (26). Then the obtained expression is transformed similarly to the series (19). In the result, the following expansion is developed

$$
\sin ^{2} \theta T_{y y}^{(1)}=\sum_{|m|=0}^{1} \sum_{n=|m|}^{N+2} \bar{D}_{n, m} \bar{Y}_{n, m}
$$

where

$$
\begin{aligned}
& \sum_{|m|=0}^{1} \sum_{n=|m|}^{N+2} \bar{D}_{n, m} \bar{Y}_{n, m}=-\sum_{|m|=0}^{1} \sum_{n=4}^{N+2} \bar{a}_{n, m} \bar{G}_{n-2, m} \bar{Y}_{n, m}+\sum_{|m|=0}^{1} \sum_{n=2}^{N} \bar{b}_{n, m} \bar{G}_{n, m} \bar{Y}_{n, m} \\
& -\sum_{|m|=0}^{1} \sum_{n=2}^{N-2} \bar{c}_{n, m} \bar{G}_{n+2, m} \bar{Y}_{n, m}+\left(\frac{2}{3} \bar{G}_{0,0}-\frac{2}{3 \sqrt{5}} \bar{G}_{2,0}\right) \bar{Y}_{0,0}+\left(\frac{2}{5} \bar{G}_{1,0}-\frac{2 \sqrt{3}}{5 \sqrt{7}} \bar{G}_{3,0}\right) \bar{Y}_{1,0} \\
& +\left(\frac{4}{5} \bar{G}_{1,1}-\frac{2 \sqrt{2}}{5 \sqrt{7}} \bar{G}_{3,1}\right) \bar{Y}_{1,1}+\left(\frac{4}{5} \bar{G}_{1,-1}-\frac{2 \sqrt{2}}{5 \sqrt{7}} \bar{G}_{3,-1}\right) \bar{Y}_{1,-1}-\frac{2}{3 \sqrt{5}} \bar{G}_{0,0} \bar{Y}_{2,0} \\
& -\frac{2 \sqrt{3}}{5 \sqrt{7}} \bar{G}_{1,0} \bar{Y}_{3,0}-\frac{2 \sqrt{2}}{5 \sqrt{7}} \bar{G}_{1,1} \bar{Y}_{3,1}-\frac{2 \sqrt{2}}{5 \sqrt{7}} \bar{G}_{1,-1} \bar{Y}_{3,-1} .
\end{aligned}
$$

Since expression (21) does not contain the harmonics of the zero and first degrees then in (30) such harmonics have also to be absent. It means that the quantities inside the brackets in (30) are equal to zero and therefore

$$
\bar{G}_{0,0}=\frac{1}{\sqrt{5}} \bar{G}_{2,0}, \bar{G}_{1,0}=\sqrt{\frac{3}{7}} \bar{G}_{3,0}, \bar{G}_{1, m}=\frac{1}{\sqrt{14}} \bar{G}_{3, m},|m|=1 .
$$

We substitute the expressions from (31) into the last four terms of (30) and then add these transformed terms to the second sum in the right-hand side of equation (30). 
Since the right-hand side of equation (30) does not contain $\bar{G}_{N+1, m}$ and $\bar{G}_{N+2, m}$ then we have in the series (25) and (8)

$$
\bar{G}_{N+1, m}=\bar{G}_{N+2, m}=0,|m|=0,1 .
$$

We have derived two expressions for the same function $\sin ^{2} \theta T_{y y}^{(1)}$, one being defined by equations (20) and (21), for $|m|=0,1$, and the other by equation (29) and the transformed equation (30). Therefore the coefficients of the spherical harmonics of the same order $m$ and degree $n$ in (21) and (30)-(32) are equal to each other.

After equating the harmonic coefficients from both sides of equation (21) the following set of equations is obtained

$$
\left.\begin{array}{rl}
-\bar{h}_{\bar{m}, m} \bar{R}_{\bar{m}, m} & =\bar{D}_{\bar{m}, m} \\
-\bar{h}_{\bar{m}+1, m} \bar{R}_{\bar{m}+1, m} & =\bar{D}_{\bar{m}+1, m} \\
\bar{g}_{\bar{m}+2, m} \bar{R}_{\bar{m}, m}-\bar{h}_{\bar{m}+2, m} \bar{R}_{\bar{m}+2, m}=\bar{D}_{\bar{m}+2, m} \\
\bar{g}_{\bar{m}+3, m} \bar{R}_{\bar{m}+1, m}-\bar{h}_{\bar{m}+3, m} \bar{R}_{\bar{m}+3, m}=\bar{D}_{\bar{m}+3, m} \\
\ldots \\
\bar{g}_{N-2, m} \bar{R}_{N-4, m}-\bar{h}_{N-2, m} \bar{R}_{N-2, m}=\bar{D}_{N-2, m} \\
\bar{g}_{N-1, m} \bar{R}_{N-3, m}-\bar{h}_{N-1, m} \bar{R}_{N-1, m}=\bar{D}_{N-1, m} \\
\bar{g}_{N, m} \bar{R}_{N-2, m}-\bar{h}_{N, m} \bar{R}_{N, m}=\bar{D}_{N, m} \\
\bar{g}_{N+1, m} \bar{R}_{N-1, m}=\bar{D}_{N+1, m} \\
\bar{g}_{N+2, m} \bar{R}_{N, m} & =\bar{D}_{N+2, m} \\
|m|=0,1, \ldots, N, \bar{m} & =\max \{2,|m|\} .
\end{array}\right\}
$$

By equating the harmonic coefficients from both sides of the transformed equation (30) the set of equations is derived

$$
\left.\begin{array}{rl}
\bar{b}_{2, m}^{*} \bar{G}_{2, m}-\bar{c}_{2, m} \bar{G}_{4, m} & =\bar{D}_{2, m} \\
\bar{b}_{3, m}^{*} \bar{G}_{3, m}-\bar{c}_{3, m} \bar{G}_{5, m} & =\bar{D}_{3, m} \\
-\bar{a}_{4, m} \bar{G}_{2, m}+\bar{b}_{4, m}^{*} \bar{G}_{4, m}-\bar{c}_{4, m} \bar{G}_{6, m} & =\bar{D}_{4, m} \\
-\bar{a}_{5, m} \bar{G}_{3, m}+\bar{b}_{5, m}^{*} \bar{G}_{5, m}-\bar{c}_{5, m} \bar{G}_{7, m} & =\bar{D}_{5, m} \\
& \cdots \\
-\bar{a}_{N-2, m} \bar{G}_{N-4, m}+\bar{b}_{N-2, m}^{*} \bar{G}_{N-2, m}-\bar{c}_{N-2, m} \bar{G}_{N, m} & =\bar{D}_{N-2, m} \\
-\bar{a}_{N-1, m} \bar{G}_{N-3, m}+\bar{b}_{N-1, m}^{*} \bar{G}_{N-1, m} & =\bar{D}_{N-1, m} \\
-\bar{a}_{N, m} \bar{G}_{N-2, m}+\bar{b}_{N, m}^{*} \bar{G}_{N, m} & =\bar{D}_{N, m} \\
-\bar{a}_{N+1, m} \bar{G}_{N-1, m} & =\bar{D}_{N+1, m} \\
-\bar{a}_{N+2, m} \bar{G}_{N, m} & =\bar{D}_{N+2, m} \\
|m| & =0,1 .
\end{array}\right\}
$$


Equations (33), taken for $|m|=0,1$, and equations (34) are considered together and applied for solving two inverse problems.

Let us assume that a geopotential model represented by the series (1) is known. Then, on the basis of the quantities $\bar{R}_{n, m}$ defined in (6), the coefficients $\bar{G}_{n, m}$ of the series (8) can be determined for $|m|=0,1$ in the following manner.

From (33) the quantities $\bar{D}_{n, m}$ are evaluated for $|m|=0,1$ on the basis of $\bar{R}_{n, m}$. We disregard the first two equations in (34). After substituting in the right-hand sides the known quantities $\bar{D}_{n, m}$, we solve the remaining set of equations (34) with respect to $\bar{G}_{n, m}$ for $n=2,3, \ldots, N$ by a recurrent procedure, beginning from the last two equations. After solving equations (34), the coefficients $\bar{G}_{n, m}$ in (25) are found for $n=0,1$ from equations (31). The additional equations (32) are also used. Thus, all the coefficients $\bar{G}_{n, m}$ in Eq. (25) are determined.

Let us suppose now that from gradiometry data the coefficients $\bar{G}_{n, m}$ are evaluated for $|m|=0,1$ and $n=2,3, \ldots, N$. Then from equations (34) the corresponding quantities $\bar{D}_{n, m}$ can be estimated. We disregard in (33) the first two equations and substitute in the remaining equations the known quantities $\bar{D}_{n, m}$ for $|m|=0,1$. Then from equations (33) the coefficients $\bar{R}_{n, m}$ are calculated for $|m|=0,1$ and $n=2,3, \ldots, N$ by a recurrent procedure, beginning from the last two equations. From the quantities $\bar{R}_{n, m}$ the coefficients $\bar{C}_{n, m}$ are found, according to (6). In this way a geopotential model defined in (1) is constructed for $|m|=0,1$.

Let us consider the function $T_{y y}^{(2)}$ represented by equation (24). This expression is transformed in the series of the modified spherical harmonics, defined in (8) and (12),

$$
T_{y y}^{(2)}=\sum_{|m|=2}^{N} \sum_{n=|m|}^{N+2} \bar{G}_{n, m} \bar{P}_{n-2,|m|-2}(\cos \theta) Q_{m}(\lambda) .
$$

The series (35) is presented in the non-normalized form and then multiplied by $\sin ^{2} \theta$. It gives

$$
\sin ^{2} \theta T_{y y}^{(2)}=\sum_{|m|=2}^{N} \sum_{n=|m|}^{N+2} \sigma_{n,|m|} G_{n, m} \sin ^{2} \theta P_{n-2,|m|-2}(\cos \theta) Q_{m}(\lambda) .
$$

The right-hand side of (36) is divided in two sums

$$
\begin{aligned}
\sin ^{2} \theta T_{y y}^{(2)}= & \sum_{|m|=2}^{3} \sum_{n=|m|}^{3} \sigma_{n,|m|} G_{n, m} \sin ^{2} \theta P_{n-2,|m|-2}(\cos \theta) Q_{m}(\lambda) \\
& +\sum_{|m|=2}^{N} \sum_{n=\max \{4,|m|\}}^{N+2} \sigma_{n,|m|} G_{n, m} \sin ^{2} \theta P_{n-2,|m|-2}(\cos \theta) Q_{m}(\lambda)
\end{aligned}
$$

From equation (1.61) in Heiskanen and Moritz (1979) we have 


$$
\begin{aligned}
& \sin ^{2} \theta P_{0,0}(\cos \theta)=\frac{1}{3} P_{2,2}(\cos \theta), \quad \sin ^{2} \theta P_{1,0}(\cos \theta)=\frac{1}{15} P_{3,2}(\cos \theta), \\
& \sin ^{2} \theta P_{1,1}(\cos \theta)=\frac{1}{15} P_{3,3}(\cos \theta) .
\end{aligned}
$$

In Claessens (2005, Table 2) there is the relation

$$
\begin{aligned}
\sin ^{2} \theta P_{n,|m|} & =\frac{1}{(2 n+1)(2 n+3)} P_{n+2,|m|+2} \\
& -\frac{2}{(2 n-1)(2 n+3)} P_{n,|m|+2}+\frac{1}{(2 n-1)(2 n+1)} P_{n-2,|m|+2} .
\end{aligned}
$$

Expressions (38) are applied to the first double sum from (37) and equation (39) to the second one. Then the obtained expression in (37) is transformed in a manner described after equation (19).

In the result, the following spherical harmonic series is derived

$$
\sin ^{2} \theta T_{y y}^{(2)}=\sum_{|m|=2}^{N} \sum_{n=|m|}^{N+2} \bar{D}_{n, m} \bar{Y}_{n, m}(\theta, \lambda),
$$

where

$$
\begin{aligned}
& \sum_{|m|=2}^{N} \sum_{n=|m|}^{N+2} \bar{D}_{n, m} \bar{Y}_{n, m}(\theta, \lambda)=\sum_{|m|=2}^{N} \sum_{n=|m|}^{N+2} \bar{a}_{n, m} \bar{G}_{n, m} \bar{Y}_{n, m}(\theta, \lambda) \\
& +\sum_{|m|=2}^{N} \sum_{n=|m|}^{N} \bar{b}_{n, m} \bar{G}_{n+2, m} \bar{Y}_{n, m}(\theta, \lambda)+\sum_{|m|=2}^{N-2} \sum_{n=|m|}^{N-2} \bar{c}_{n, m} \bar{G}_{n+4, m} \bar{Y}_{n, m}(\theta, \lambda) .
\end{aligned}
$$

From (41) the set of equations follows

$$
\left.\begin{array}{rl}
\bar{a}_{\bar{m}, m} \bar{G}_{\bar{m}, m}+\bar{b}_{\bar{m}, m} \bar{G}_{\bar{m}+2, m}+\bar{c}_{\bar{m}, m} \bar{G}_{\bar{m}+4, m}=\bar{D}_{\bar{m}, m} \\
\bar{a}_{\bar{m}+1, m} \bar{G}_{\bar{m}+1, m}+\bar{b}_{\bar{m}+1, m} \bar{G}_{\bar{m}+3, m}+\bar{c}_{\bar{m}+1, m} \bar{G}_{\bar{m}+5, m}=\bar{D}_{\bar{m}+1, m} \\
\ldots \\
\bar{a}_{N-2, m} \bar{G}_{N-2, m}+\bar{b}_{N-2, m} \bar{G}_{N, m}+\bar{c}_{N-2, m} \bar{G}_{N+2, m}=\bar{D}_{N-2, m} \\
\bar{a}_{N-1, m} \bar{G}_{N-1, m}+\bar{b}_{N-1, m} \bar{G}_{N+1, m}=\bar{D}_{N-1, m} \\
\bar{a}_{N, m} \bar{G}_{N, m}+\bar{b}_{N, m} \bar{G}_{N+2, m}=\bar{D}_{N, m} \\
\bar{a}_{N+1, m} \bar{G}_{N+1, m}=\bar{D}_{N+1, m} \\
\bar{a}_{N+2, m} \bar{G}_{N+2, m}=\bar{D}_{N+2, m} \\
|m|=2,3, \ldots, N, \bar{m}=|m|
\end{array}\right\}
$$

Equations (33), for $|m|=2,3, \ldots, N$, and equations (42) are applied for solving two inverse problems similar to the case when $|m|=0,1$. 


\subsection{DERIVATIVE $T_{x x}$}

Let us consider the derivative $T_{x x}$. We shall not perform transformations similar to those developed for $T_{y y}$, but apply a more simple approach based on the Laplace equation

$$
T_{x x}+T_{y y}+T_{z z}=0
$$

Function $T_{x x}$ is presented in the form

$$
T_{x x}=T_{x x}^{(1)}+T_{x x}^{(2)}
$$

where $T_{x x}^{(1)}$ corresponds to $|m|=0,1$ and $T_{x x}^{(2)}$ to $|m|=2,3, \ldots, N$.

From expansion (5) follows

$$
T_{z z}^{(1)}=\sum_{|m|=0}^{1} \sum_{n=2}^{N} \bar{R}_{n, m} \bar{P}_{n,|m|}(\cos \theta) Q_{m}(\lambda) .
$$

From equations (25), (32), (43) and (45) we obtain

$$
T_{x x}^{(1)}=-\sum_{|m|=0}^{1} \sum_{n=|m|}^{N} \bar{G}_{n, m} \bar{P}_{n, m}(\cos \theta) Q_{m}(\lambda)-\sum_{|m|=0}^{1} \sum_{n=2}^{N} \bar{R}_{n, m} \bar{P}_{n, m}(\cos \theta) Q_{m}(\lambda) .
$$

This expression is presented in the form

$$
T_{x x}^{(1)}=\sum_{|m|=0}^{1} \sum_{n=|m|}^{N} \bar{H}_{n, m} \bar{P}_{n, m}(\cos \theta) Q_{m}(\lambda) .
$$

From (47) follows that in the series (7)

$$
\bar{H}_{N+1, m}=\bar{H}_{N+2, m}=0,|m|=0,1 .
$$

From (46) and (47) it is obtained

$$
\bar{H}_{0,0}=-\bar{G}_{0,0}, \bar{H}_{1,0}=-\bar{G}_{1,0}, \bar{H}_{1, m}=-\bar{G}_{1, m},|m|=0,1
$$

and

$$
\bar{H}_{n, m}=-\bar{G}_{n, m}-\bar{R}_{n, m},|m|=0,1, n=2,3, \ldots, N .
$$

If the coefficients $\bar{G}_{n, m}$ have been synthesized from a geopotential model, as it is described in Sec. 3.1, then the coefficients $\bar{H}_{n, m}$ in (7) can be synthesized by applying equations (49) and (50).

Let us solve the problem of constructing a geopotential model on the basis of known $\bar{H}_{n, m}$.

It is supposed that the coefficients $\bar{H}_{n, m}$ in (7) are determined from gradiometry data. Then from (50) follows

$$
\bar{G}_{n, m}=-\bar{R}_{n, m}-\bar{H}_{n, m},|m|=0,1, n=2,3, \ldots, N .
$$


For $|m|=0,1$ we apply together equations (33), (34) and (51). The expressions from (51) are substituted in the left-hand sides of (34). At the same time, we substitute the expressions for $\bar{D}_{n, m}$ from (33), for $|m|=0,1$, in the right-hand sides of (34).

After combining together in the transformed equations (34) the terms depending on $\bar{R}_{n, m}$, in the left-hand sides, and those depending on $\bar{H}_{n, m}$, in the right-hand sides, we obtain the following sets of equations, for $|m|=0,1$,

$$
\left.\begin{array}{rl}
\hat{b}_{2, m} \bar{R}_{2, m}-\bar{c}_{2, m} \bar{R}_{4, m} & =\bar{K}_{2, m} \\
\hat{b}_{3, m} \bar{R}_{3, m}-\bar{c}_{3, m} \bar{R}_{5, m} & =\bar{K}_{3, m} \\
-\hat{a}_{4, m} \bar{R}_{2, m}+\hat{b}_{4, m} \bar{R}_{4, m}-\bar{c}_{4, m} \bar{R}_{6, m} & =\bar{K}_{4, m} \\
-\hat{a}_{5, m} \bar{R}_{3, m}+\hat{b}_{5, m} \bar{R}_{5, m}-\bar{c}_{5, m} \bar{R}_{7, m} & =\bar{K}_{5, m} \\
\cdots & \\
-\hat{a}_{N-2, m} \bar{R}_{N-4, m}+\hat{b}_{N-2, m} \bar{R}_{N-2, m}-\bar{c}_{N-2, m} \bar{R}_{N, m} & =\bar{K}_{N-2, m} \\
\hat{a}_{N-1, m} \bar{R}_{N-3, m}+\hat{b}_{N-1, m} \bar{R}_{N-1, m} & =\bar{K}_{N-1, m} \\
-\hat{a}_{N, m} \bar{R}_{N-2, m}+\hat{b}_{N, m} \bar{R}_{N, m} & =\bar{K}_{N, m} \\
-\hat{a}_{N+1, m} \bar{R}_{N-1, m} & =\bar{K}_{N+1, m} \\
-\hat{a}_{N+2, m} \bar{R}_{N, m} & =\bar{K}_{N+2, m} \\
|m| & =0,1
\end{array}\right\}
$$

and

$$
\left.\begin{array}{rl}
-\bar{b}_{2, m}^{*} \bar{H}_{2, m}+\bar{c}_{2, m} \bar{H}_{4, m} & =\bar{K}_{2, m} \\
-\bar{b}_{3, m}^{*} \bar{H}_{3, m}+\bar{c}_{3, m} \bar{H}_{5, m} & =\bar{K}_{3, m} \\
\bar{a}_{4, m} \bar{H}_{2, m}-\bar{b}_{4, m}^{*} \bar{H}_{4, m}+\bar{c}_{4, m} \bar{H}_{6, m} & =\bar{K}_{4, m} \\
\bar{a}_{5, m} \bar{H}_{3, m}-\bar{b}_{5, m}^{*} \bar{H}_{5, m}+\bar{c}_{5, m} \bar{H}_{7, m} & =\bar{K}_{5, m} \\
& \cdots \\
\bar{a}_{N-2, m} \bar{H}_{N-4, m}-\bar{b}_{N-2, m}^{*} \bar{H}_{N-2, m}+\bar{c}_{N-2, m} \bar{H}_{N, m} & =\bar{K}_{N-2, m} \\
\bar{a}_{N-1, m} \bar{H}_{N-3, m}-\bar{b}_{N-1, m}^{*} \bar{H}_{N-1, m} & =\bar{K}_{N-1, m} \\
\bar{a}_{N, m} \bar{H}_{N-2, m}-\bar{b}_{N, m}^{*} \bar{H}_{N, m} & =\bar{K}_{N, m} \\
\bar{a}_{N+1, m} \bar{H}_{N-1, m} & =\bar{K}_{N+1, m} \\
\bar{a}_{N+2, m} \bar{H}_{N, m} & =\bar{K}_{N+2, m} \\
|m| & =0,1 .
\end{array}\right\}
$$

From (53) the quantities $\bar{K}_{n, m}$ can be evaluated on the basis of known $\bar{H}_{n, m}$ and then substituted in (52). We disregard in (52) the first two equations and solve the remaining equations with respect to the unknown $\bar{R}_{n, m}$ by a recurrent procedure, beginning from the last 
two equations. After applying expression (6), the coefficients $\bar{C}_{n, m}$ are determined for $|m|=0,1$.

Let us consider the case when $|m|=2,3, \ldots, N$.

From (43) follows

$$
\sin ^{2} \theta T_{x x}^{(2)}=-\sin ^{2} \theta T_{y y}^{(2)}-\sin ^{2} \theta T_{z z}^{(2)} .
$$

The expression for $\sin ^{2} \theta T_{y y}^{(2)}$ is derived from (20) and (21), for $|m|=2,3, \ldots, N$, and then substituted in (54).

From (6) we have

$$
\sin ^{2} \theta T_{z z}^{(2)}=\sum_{|m|=2}^{N} \sum_{n=|m|}^{N} R_{n, m} \sin ^{2} \theta P_{n,|m|}(\cos \theta) Q_{m}(\lambda) .
$$

We apply expression (28) with respect to the right-hand side of (55). The resulting equation is transformed in the same manner, as it is described after equation (19), and then substituted in (54).

In the result of the above transformations of the right-hand side of (54) the following expansion is derived

$$
\sin ^{2} \theta T_{x x}^{(2)}=\sum_{|m|=2}^{N} \sum_{n=|m|}^{N+2} \bar{K}_{n, m} \bar{Y}_{n, m}(\theta, \lambda)
$$

where

$$
\begin{aligned}
& \sum_{|m|=2}^{N} \sum_{n=|m|}^{N+2} \bar{K}_{n, m} \bar{Y}_{n, m}(\theta, \lambda)=\sum_{|m|=2}^{N} \sum_{n=|m|+2}^{N+2} p_{n, m} \bar{R}_{n-2, m} \bar{Y}_{n, m}(\theta, \lambda) \\
& +\sum_{|m|=2}^{N} \sum_{n=|m|}^{N} q_{n, m} \bar{R}_{n, m} \bar{Y}_{n, m}(\theta, \lambda)+\sum_{|m|=2}^{N-2} \sum_{n=|m|}^{N-2} r_{n, m} \bar{R}_{n+2, m} \bar{Y}_{n, m}(\theta, \lambda) .
\end{aligned}
$$

From (57) the set of equations follows

$$
\left.\begin{array}{rl}
q_{\bar{m}, m} \bar{R}_{\bar{m}, m}+r_{\bar{m}, m} \bar{R}_{\bar{m}+2, m} & =\bar{K}_{\bar{m}, m} \\
q_{\bar{m}+1, m} \bar{R}_{\bar{m}+1, m}+r_{\bar{m}+3, m} \bar{R}_{\bar{m}+3, m} & =\bar{K}_{\bar{m}+1, m} \\
p_{\bar{m}+2, m} \bar{R}_{\bar{m}, m}+q_{\bar{m}+2, m} \bar{R}_{\bar{m}+2, m}+r_{\bar{m}+2, m} \bar{R}_{\bar{m}+4, m} & =\bar{K}_{\bar{m}+2, m} \\
p_{\bar{m}+3, m} \bar{R}_{\bar{m}+1, m}+q_{\bar{m}+3, m} \bar{R}_{\bar{m}+3, m}+r_{\bar{m}+3, m} \bar{R}_{\bar{m}+5, m} & =\bar{K}_{\bar{m}+3, m} \\
& \cdots \\
p_{N-2, m} \bar{R}_{N-4, m}+q_{N-2, m} \bar{R}_{N-2, m}+r_{N-2, m} \bar{R}_{N, m} & =\bar{K}_{N-2, m} \\
p_{N-1, m} \bar{R}_{N-3, m}+q_{N-1, m} \bar{R}_{N-1, m} & =\bar{K}_{N-1, m} \\
p_{N, m} \bar{R}_{N-2, m}+q_{N, m} \bar{R}_{N, m} & =\bar{K}_{N, m} \\
p_{N+1, m} \bar{R}_{N-1, m} & =\bar{K}_{N+1, m} \\
p_{N+2, m} \bar{R}_{N, m} & =\bar{K}_{N+2, m} \\
|m|=2,3, \ldots, N, \bar{m} & =\mid m .
\end{array}\right\}
$$


According to (7) and (12), we present the function $T_{x x}^{(2)}$ in the form

$$
T_{x x}^{(2)}=\sum_{|m|=2}^{N} \sum_{n=|m|}^{N+2} \bar{H}_{n, m} \bar{P}_{n-2,|m|-2}(\cos \theta) Q_{m}(\lambda) .
$$

We can observe that equations (59) and (56) have the same form as equations (35) and (40). Then, after changing $\bar{G}_{n, m}$ for $\bar{H}_{n, m}$ and $\bar{D}_{n, m}$ for $\bar{K}_{n, m}$, we can derive equations for $T_{x x}^{(2)}$ similar to (40)-(42). In the result, a set of equations, similar to (42), are obtained

$$
\left.\begin{array}{rl}
\bar{a}_{\bar{m}, m} \bar{H}_{\bar{m}, m}+\bar{b}_{\bar{m}, m} \bar{H}_{\bar{m}+2, m}+\bar{c}_{\bar{m}, m} \bar{H}_{\bar{m}+4, m}=\bar{K}_{\bar{m}, m} \\
\bar{a}_{\bar{m}+1, m} \bar{H}_{\bar{m}+1, m}+\bar{b}_{\bar{m}+1, m} \bar{H}_{\bar{m}+3, m}+\bar{c}_{\bar{m}+1, m} \bar{H}_{\bar{m}+5, m}=\bar{K}_{\bar{m}+1, m} \\
\bar{a}_{N-2, m} \bar{H}_{N-2, m}+\bar{b}_{N-2, m} \bar{H}_{N, m}+\bar{c}_{N-2, m} \bar{H}_{N+2, m}=\bar{K}_{N-2, m} \\
\bar{a}_{N-1, m} \bar{H}_{N-1, m}+\bar{b}_{N-1, m} \bar{H}_{N+1, m}=\bar{K}_{N-1, m} \\
\bar{a}_{N, m} \bar{H}_{N, m}+\bar{b}_{N, m} \bar{H}_{N+2, m}=\bar{K}_{N, m} \\
\bar{a}_{N+1, m} \bar{H}_{N+1, m}=\bar{K}_{N+1, m} \\
\bar{a}_{N+2, m} \bar{H}_{N+2, m}=\bar{K}_{N+2, m} \\
|m|=2,3, \ldots, N, \bar{m}=|m| .
\end{array}\right\}
$$

The sets of equations (58) and (60) can be applied for recovering $\bar{R}_{n, m}$ from $\bar{H}_{n, m}$. Then from (6) the coefficients $\bar{C}_{n, m}$ will be determined for $|m|=2,3, \ldots, N$.

\subsection{DERIVATIVE $T_{x y}$}

Let us construct the series (9) for $T_{x y}$.

After applying equation (6), we present equation (30) from PV2006 in the form

$$
\begin{aligned}
\sin ^{2} \theta T_{x y}= & \sum_{|m|=1}^{N} \sum_{n=|m|}^{N} R_{n,-m} Q_{m}(\lambda) \\
& \times\left[\frac{m}{(2 n+1)} \frac{(n-1)(n-|m|+1)}{(n+1)(n+2)} P_{n+1,|m|}(\cos \theta)-\frac{(n+|m|)}{(n+1)} P_{n-1,|m|}(\cos \theta)\right] .
\end{aligned}
$$

Expression (61) is transformed similarly to expression (19) for $\sin ^{2} \theta T_{y y}$. In the result, the following equation is derived

$$
\sin ^{2} \theta T_{x y}=\sum_{|m|=1}^{N} \sum_{n=|m|}^{N+1} \bar{L}_{n, m} \bar{Y}_{n, m}(\theta, \lambda),
$$

where

$$
\begin{aligned}
\sum_{|m|=1}^{N} \sum_{n=|m|}^{N+1} \bar{L}_{n, m} \bar{Y}_{n, m}(\theta, \lambda)= & \sum_{|m|=1}^{N} \sum_{n=\max \{3,|m|+1\}}^{N+1} \bar{\xi}_{n, m} \bar{R}_{n-1,-m} \bar{Y}_{n, m}(\theta, \lambda) \\
& -\sum_{|m|=1}^{N-1} \sum_{n=|m|}^{N-1} \bar{\eta}_{n, m} \bar{R}_{n+1,-m} \bar{Y}_{n, m}(\theta, \lambda) .
\end{aligned}
$$


Function $T_{x y}$ is presented as the sum

$$
T_{x y}=T_{x y}^{(1)}+T_{x y}^{(2)}
$$

where the first component corresponds to $|m|=1$ and the second one to $|m|=2,3, \ldots, N$.

According to (9) and (12), the following expansion is constructed

$$
T_{x y}^{(1)}=\sum_{|m|=1} \sum_{n=1}^{N+1} \bar{M}_{n, m} \bar{P}_{n,|m|}(\cos \theta) Q_{m}(\lambda) .
$$

After transferring to the non-normalized quantities, this equation is multiplied by $\sin ^{2} \theta$ and then the obtained expression is transformed similarly to expression (19). In the result, the equation is derived

$$
\sin ^{2} \theta T_{x y}^{(1)}=\sum_{|m|=1} \sum_{n=1}^{N+1} \bar{L}_{n, m} \bar{Y}_{n, m}(\theta, \lambda)
$$

where

$$
\begin{aligned}
\sum_{|m|=1} \sum_{n=1}^{N+1} \bar{L}_{n, m} \bar{Y}_{n, m}(\theta, \lambda)= & -\sum_{|m|=1} \sum_{n=4}^{N+1} \bar{a}_{n, m} \bar{M}_{n-2, m} \bar{Y}_{n, m}(\theta, \lambda)+\sum_{|m|=1} \sum_{n=2}^{N-1} \bar{b}_{n, m} \bar{M}_{n, m} \bar{Y}_{n, m}(\theta, \lambda) \\
& -\sum_{|m|=1} \sum_{n=2}^{N-3} \bar{c}_{n, m} \bar{M}_{n+2, m} \bar{Y}_{n, m}(\theta, \lambda)+\left(\frac{4}{5} \bar{M}_{1,1}-\frac{2 \sqrt{2}}{5 \sqrt{7}} \bar{M}_{3,1}\right) \bar{Y}_{1,1} \\
& +\left(\frac{4}{5} \bar{M}_{1,-1}-\frac{2 \sqrt{2}}{5 \sqrt{7}} \bar{M}_{3,-1}\right) \bar{Y}_{1,-1}-\frac{2 \sqrt{2}}{5 \sqrt{7}} \bar{M}_{1,1} \bar{Y}_{3,1}-\frac{2 \sqrt{2}}{5 \sqrt{7}} \bar{M}_{1,-1} \bar{Y}_{3,-1} .
\end{aligned}
$$

Since the right-hand side of (67) does not contain $\bar{M}_{N, m}$ and $\bar{M}_{N+1, m}$ then in (9) and (65) we have

$$
\bar{M}_{N, m}=\bar{M}_{N+1, m}=0,|m|=1 .
$$

From (63) the set of equations follows

$$
\left.\begin{array}{rl}
-\bar{\eta}_{\bar{m}, m} \bar{R}_{\bar{m}+1,-m} & =\bar{L}_{\bar{m}, m} \\
\bar{\xi}_{\bar{m}+1, m} \bar{R}_{\bar{m},-m}-\bar{\eta}_{\bar{m}+1, m} \bar{R}_{\bar{m}+2,-m} & =\bar{L}_{\bar{m}+1, m} \\
\bar{\xi}_{\bar{m}+2, m} \bar{R}_{\bar{m}+1,-m}-\bar{\eta}_{\bar{m}+2, m} \bar{R}_{\bar{m}+3,-m} & =\bar{L}_{\bar{m}+2, m}, \\
\ldots & \\
\bar{\xi}_{N-2, m} \bar{R}_{N-3,-m}-\bar{\eta}_{N-2, m} \bar{R}_{N-1,-m} & =\bar{L}_{N-2, m} \\
\bar{\xi}_{N-1, m} \bar{R}_{N-2,-m}-\bar{\eta}_{N-1, m} \bar{R}_{N,-m} & =\bar{L}_{N-1, m} \\
\bar{\xi}_{N, m} \bar{R}_{N-1,-m} & =\bar{L}_{N, m} \\
\bar{\xi}_{N+1, m} \bar{R}_{N,-m} & =\bar{L}_{N+1, m} \\
|m|=1,2, \ldots, N, \bar{m} & =|m| .
\end{array}\right\}
$$

On the basis of (67) we derive the set of equations 


$$
\left.\begin{array}{rl}
\bar{b}_{1, m} \bar{M}_{1, m}-\bar{c}_{1, m} \bar{M}_{3, m} & =\bar{L}_{1, m} \\
\bar{b}_{2, m} \bar{M}_{2, m}-\bar{c}_{2, m} \bar{M}_{4, m} & =\bar{L}_{2, m} \\
-\bar{a}_{3, m} \bar{M}_{1, m}+\bar{b}_{3, m} \bar{M}_{3, m}-\bar{c}_{3, m} \bar{M}_{5, m} & =\bar{L}_{3, m} \\
-\bar{a}_{4, m} \bar{M}_{2, m}+\bar{b}_{4, m} \bar{M}_{4, m}-\bar{c}_{4, m} \bar{M}_{6, m} & =\bar{L}_{4, m} \\
& \cdots \\
-\bar{a}_{N-3, m} \bar{M}_{N-5, m}+\bar{b}_{N-3, m} \bar{M}_{N-3, m}-\bar{c}_{N-3, m} \bar{M}_{N-1, m} & =\bar{L}_{N-3, m} \\
-\bar{a}_{N-2, m} \bar{M}_{N-4, m}+\bar{b}_{N-2, m} \bar{M}_{N-2, m} & =\bar{L}_{N-2, m} \\
-\bar{a}_{N-1, m} \bar{M}_{N-3, m}+\bar{b}_{N-1, m} \bar{M}_{N-1, m} & =\bar{L}_{N-1, m} \\
-\bar{a}_{N, m} \bar{M}_{N-2, m} & =\bar{L}_{N, m} \\
-\bar{a}_{N+1, m} \bar{M}_{N-1, m} & =\bar{L}_{N+1, m} \\
|m| & =1 .
\end{array}\right\}
$$

In (69) the first equation is disregarded. Then equations (69), for $|m|=1$, and equations (70) can be applied for solving two inverse problems, for $|m|=1$, as in the cases of $T_{x x}$ and $T_{y y}$.

For $T_{x y}^{(2)}$, defined in (9) and (12), we have

$$
T_{x y}^{(2)}=\sum_{|m|=2}^{N} \sum_{n=|m|}^{N+1} \bar{M}_{n, m} \bar{P}_{n-2,|m|-2}(\cos \theta) Q_{m}(\lambda) .
$$

Expression (71) differs from expression (35) for $T_{y y}^{(2)}$ only by the upper limit for $n$. Consequently, for function $T_{x y}^{(2)}$ we can write equations similar to equations (40)-(42). In the result, the following set of equations is obtained

$$
\left.\begin{array}{rl}
\bar{a}_{\bar{m}, m} \bar{M}_{\bar{m}, m}+\bar{b}_{\bar{m}, m} \bar{M}_{\bar{m}+2, m}+\bar{c}_{\bar{m}, m} \bar{M}_{\bar{m}+4, m} & =\bar{L}_{\bar{m}, m} \\
\bar{a}_{\bar{m}+1, m} \bar{M}_{\bar{m}+1, m}+\bar{b}_{\bar{m}+1, m} \bar{M}_{\bar{m}+3, m}+\bar{c}_{\bar{m}+1, m} \bar{M}_{\bar{m}+5, m} & =\bar{L}_{\bar{m}+1, m} \\
\ldots & \\
\bar{a}_{N-3, m} \bar{M}_{N-3, m}+\bar{b}_{N-3, m} \bar{M}_{N-1, m}+\bar{c}_{N-3, m} \bar{M}_{N+1, m} & =\bar{L}_{N-3, m} \\
\bar{a}_{N-2, m} \bar{M}_{N-2, m}+\bar{b}_{N-2, m} \bar{M}_{N, m} & =\bar{L}_{N-2, m} \\
\bar{a}_{N-1, m} \bar{M}_{N-1, m}+\bar{b}_{N-1, m} \bar{M}_{N+1, m} & =\bar{L}_{N-1, m} \\
\bar{a}_{N, m} \bar{M}_{N, m} & =\bar{L}_{N, m} \\
\bar{a}_{N+1, m} \bar{M}_{N+1, m} & =\bar{L}_{N+1, m} \\
|m|=2,3, \ldots, N, \bar{m} & =|m| .
\end{array}\right\}
$$

In (70) we disregard the first two equations. Then from equations (69), (70) and (72) two inverse problems can be solved. 


\subsection{DERIVATIVE $T_{x z}$}

From Eqs. (36), (38) and (39) in PV2006 follows

$$
T_{x z}=T_{x z}^{(0)}+T_{x z}^{(1)}
$$

where

$$
T_{x z}^{(0)}=-\frac{\mu}{a^{3}} \sum_{n=2}^{N}\left(\frac{a}{r}\right)^{n+3} C_{n, 0}(n+2) P_{n, 1}(\cos \theta)
$$

and

$$
T_{x z}^{(1)}=\frac{\mu}{a^{3}} \sum_{n=2}^{N} \sum_{|m|=1}^{n}\left(\frac{a}{r}\right)^{n+3}(n+2) C_{n, m} \frac{\mathrm{d} P_{n,|m|}(\cos \theta)}{\mathrm{d} \theta} Q_{m}(\lambda) .
$$

After applying (6) we transform (74) to the form

$$
T_{x z}^{(0)}=\sum_{n=2}^{N} \bar{B}_{n, 0} \bar{P}_{n, 1}(\cos \theta)
$$

where

$$
\bar{B}_{n, 0}=-\sqrt{\frac{n}{2(n+1)}} \bar{R}_{n, 0}, n=2,3, \ldots, N .
$$

From (76) follows that in (10) we have $\bar{B}_{n, 0}=0$ for $n=0,1, N+1$.

Equation (75) is multiplied by $\sin \theta$. Then equation (Z.1.43) from Ilk (1983)

$$
\sin \theta \frac{\mathrm{d} P_{n,|m|}(\cos \theta)}{\mathrm{d} \theta}=-\frac{(n+1)(n+|m|)}{(2 n+1)} P_{n-1,|m|}(\cos \theta)+\frac{n(n-|m|+1)}{(2 n+1)} P_{n+1,|m|}(\cos \theta)
$$

is applied.

From (75), (6) and (78) the equation follows

$$
\begin{aligned}
\sin \theta T_{x z}^{(1)}= & \sum_{n=2}^{N} \sum_{|m|=1}^{n} R_{n, m} Q_{m}(\lambda)(n+2) \\
& \times\left[\frac{n(n-|m|+1)}{(2 n+1)} P_{n+1,|m|}-\frac{(n+1)(n+|m|)}{(2 n+1)} P_{n-1,|m|}\right] .
\end{aligned}
$$

We transform expression (79) in the same manner as it is described after equation (19). In the result, the following expansion is derived

$$
\sin \theta T_{x z}^{(1)}=\sum_{|m|=1}^{N} \sum_{n=|m|}^{N+1} \bar{S}_{n, m} \bar{Y}_{n,|m|}(\theta, \lambda)
$$

where 


$$
\begin{aligned}
\sum_{|m|=1}^{N} \sum_{n=|m|}^{N+1} \bar{S}_{n, m} \bar{Y}_{n,|m|}(\theta, \lambda)= & \sum_{|m|=1}^{N} \sum_{n=\max \{3,|m|+1\}}^{N+1} \bar{\beta}_{n, m} \bar{R}_{n-1, m} \bar{Y}_{n,|m|}(\theta, \lambda) \\
& -\sum_{|m|=1}^{N-1} \sum_{n=|m|}^{N-1} \bar{\gamma}_{n, m} \bar{R}_{n+1, m} \bar{Y}_{n,|m|}(\theta, \lambda) .
\end{aligned}
$$

The function $T_{x z}^{(1)}$ is presented in the form, corresponding to expressions (10) and (13):

$$
T_{x z}^{(1)}=\sum_{|m|=1}^{N} \sum_{n=|m|}^{N+1} \bar{B}_{n, m} \bar{P}_{n-1,|m|-1}(\cos \theta) Q_{m}(\lambda) .
$$

After transferring in (82) to the non-normalized quantities and multiplying this equation by $\sin \theta$ we obtain

$$
\sin \theta T_{x z}^{(1)}=\sum_{|m|=1}^{N} \sum_{n=|m|}^{N+1} \alpha_{n, m} B_{n, m} \sin \theta P_{n-1,|m|-1}(\cos \theta) Q_{m}(\lambda) .
$$

In equation (Z.1.40) from (Ilk, 1983) there is the relation

$$
\sin \theta P_{n,|m|}=\frac{1}{2 n+1}\left(P_{n+1,|m|+1}-P_{n-1,|m|+1}\right) \text {. }
$$

With taking into account (84), we can write

$$
\left.\begin{array}{c}
\sin \theta=P_{1,1}, \sin \theta P_{n-1,|m|-1}=\frac{1}{2 n-1}\left(P_{n,|m|}-P_{n-2,|m|}\right), \\
|m|=1,2, \ldots, N, \quad n=2,3, \ldots, N+1 .
\end{array}\right\}
$$

After substituting (85) in (83) the obtained equation is transformed as it is described after equation (19).

In the result, it is derived

$$
\sin \theta T_{x z}^{(1)}=\sum_{|m|=1}^{N} \sum_{n=|m|}^{N+1} \bar{S}_{n, m} \bar{Y}_{n,|m|}(\theta, \lambda)
$$

where

$$
\begin{aligned}
\sum_{|m|=1}^{N} \sum_{n=|m|}^{N+1} \bar{S}_{n, m} \bar{Y}_{n,|m|}(\theta, \lambda)= & \sum_{|m|=1}^{N} \sum_{n=|m|}^{N+1} \bar{\mu}_{n, m} \bar{B}_{n, m} \bar{Y}_{n,|m|}(\theta, \lambda) \\
& -\sum_{|m|=1}^{N-1} \sum_{n=|m|}^{N-1} \bar{v}_{n, m} \bar{B}_{n+2, m} \bar{Y}_{n,|m|}(\theta, \lambda) .
\end{aligned}
$$

From (81) and (87) two sets of equations are obtained 


$$
\left.\begin{array}{rl}
-\bar{\gamma}_{\bar{m}, m} \bar{R}_{\bar{m}+1, m} & =\bar{S}_{\bar{m}, m} \\
\bar{\beta}_{\bar{m}+1, m} \bar{R}_{\bar{m}, m}-\bar{\gamma}_{\bar{m}+1, m} \bar{R}_{\bar{m}+2, m}=\bar{S}_{\bar{m}+1, m} \\
\bar{\beta}_{\bar{m}+2, m} \bar{R}_{\bar{m}+1, m}-\bar{\gamma}_{\bar{m}+2, m} \bar{R}_{\bar{m}+3, m}=\bar{S}_{\bar{m}+2, m} \\
\ldots \\
\bar{\beta}_{N-2, m} \bar{R}_{N-3, m}-\bar{\gamma}_{N-2, m} \bar{R}_{N-1, m}=\bar{S}_{N-2, m} \\
\bar{\beta}_{N-1, m} \bar{R}_{N-2, m}-\bar{\gamma}_{N-1, m} \bar{R}_{N, m}=\bar{S}_{N-1, m} \\
\bar{\beta}_{N, m} \bar{R}_{N-1, m}=\bar{S}_{N, m} \\
\bar{\beta}_{N+1, m} \bar{R}_{N, m}=\bar{S}_{N+1, m} \\
|m|=1,2, \ldots, N, \bar{m}=|m|
\end{array}\right\}
$$

and

$$
\left.\begin{array}{rl}
\bar{\mu}_{\bar{m}, m} \bar{B}_{\bar{m}, m}-\bar{v}_{\bar{m}, m} \bar{B}_{\bar{m}+2, m}=\bar{S}_{\bar{m}, m} \\
\bar{\mu}_{\bar{m}+1, m} \bar{B}_{\bar{m}+1, m}-\bar{v}_{\bar{m}+1, m} \bar{B}_{\bar{m}+3, m}=\bar{S}_{\bar{m}+1, m} \\
\bar{\mu}_{\bar{m}+2, m} \bar{B}_{\bar{m}+2, m}-\bar{v}_{\bar{m}+2, m} \bar{B}_{\bar{m}+4, m}=\bar{S}_{\bar{m}+2, m} \\
\cdots \\
\bar{\mu}_{N-1, m} \bar{B}_{N-1, m}-\bar{v}_{N-1, m} \bar{B}_{N+1, m}=\bar{S}_{N-1, m} \\
\bar{\mu}_{N, m} \bar{B}_{N, m}=\bar{S}_{N, m} \\
\bar{\mu}_{N+1, m} \bar{B}_{N+1, m}=\bar{S}_{N+1, m} \\
|m|=1,2, \ldots, N, \bar{m}=|m| .
\end{array}\right\}
$$

The first equation in (88) is disregarded. Then equations (88) and (89) are solved similarly to the preceding cases.

\subsection{DERIVATIVE $T_{y z}$}

We apply for derivative $T_{y z}$ the expression (9) from PV2006. After transferring from $\bar{C}_{n, m}$ to the quantities $\bar{R}_{n, m}$ by means of equation (6), we obtain

$$
\sin \theta T_{y z}=\sum_{|m|=1}^{N} \sum_{n=\max \{2,|m|\}}^{N} \frac{m}{n+1} \bar{R}_{n,-m} \bar{P}_{n,|m|}(\cos \theta) Q_{m}(\lambda) .
$$

For the function $T_{y z}$ the following expansion is constructed, according to (11) and (13),

$$
T_{y z}=\sum_{|m|=1}^{N} \sum_{n=|m|}^{N} \bar{A}_{n, m} \bar{P}_{n-1,|m|-1}(\cos \theta) Q_{m}(\lambda) .
$$

Let us note that expression (91) is similar to expression (82) for $T_{x z}^{(1)}$. Only now the upper limit for $n$ is $N$ and not $N+1$. Then, after the same transformations of (91), as were applied to (82), we obtain equations similar to (83)-(87) for the function $T_{x z}^{(1)}$.

In the result, the following equation is derived 


$$
\sin \theta T_{y z}=\sum_{|m|=1}^{N} \sum_{n=|m|}^{N} \bar{\mu}_{n, m} \bar{A}_{n, m} \bar{Y}_{n,|m|}(\theta, \lambda)-\sum_{|m|=1}^{N-2} \sum_{n=|m|}^{N-2} \bar{v}_{n, m} \bar{A}_{n+2, m} \bar{Y}_{n,|m|}(\theta, \lambda)
$$

From (90) follows that in this expansion there are no harmonics of the degree $n=1$. Therefore in (92) such harmonics have to be also absent. It means that in (92)

$$
\bar{\mu}_{1,1} \bar{A}_{1, m}=\bar{v}_{1,1} \bar{A}_{3, m},|m|=1 \text {. }
$$

As a result, equation (92) becomes

$$
\sin \theta T_{y z}=\sum_{|m|=1}^{N} \sum_{n=\max \{2,|m|\}}^{N} \bar{\mu}_{n, m} \bar{A}_{n, m} \bar{Y}_{n,|m|}(\theta, \lambda)-\sum_{|m|=1}^{N-2} \sum_{n=\max \{2,|m|\}}^{N-2} \bar{v}_{n, m} \bar{A}_{n+2, m} \bar{Y}_{n,|m|}(\theta, \lambda) .
$$

By equating the right-hand sides of (90) and (94) the following set of equations is derived

$$
\left.\begin{array}{rl}
\hat{\mu}_{\bar{m}, m} \bar{A}_{\bar{m}, m}-\hat{v}_{\bar{m}, m} \bar{A}_{\bar{m}+2, m} & =\bar{R}_{\bar{m},-m} \\
\hat{\mu}_{\bar{m}+1, m} \bar{A}_{\bar{m}+1, m}-\hat{v}_{\bar{m}+1, m} \bar{A}_{\bar{m}+3, m} & =\bar{R}_{\bar{m}+1,-m} \\
\hat{\mu}_{\bar{m}+2, m} \bar{A}_{\bar{m}+2, m}-\hat{v}_{\bar{m}+2, m} \bar{A}_{\bar{m}+4, m} & =\bar{R}_{\bar{m}+2,-m} \\
\ldots & \\
\hat{\mu}_{N-2, m} \bar{A}_{N-2, m}-\hat{v}_{N-2, m} \bar{A}_{N, m} & =\bar{R}_{N-2,-m} \\
\hat{\mu}_{N-1, m} \bar{A}_{N-1, m} & =\bar{R}_{N-1,-m} \\
\hat{\mu}_{N, m} \bar{A}_{N, m} & =\bar{R}_{N,-m} \\
|m|=1,2, \ldots, N, \bar{m} & =\max \{2,|m|\} .
\end{array}\right\}
$$

It is worth noticing that equations (95) are explicitly resolved with respect to the quantities $\bar{R}_{n, m}$. Then, after applying (6), the geopotential coefficients $\bar{C}_{n, m}$ can be recovered on the basis of known spectral coefficients $\bar{A}_{n, m}$ of the derivative $T_{y z}$.

Basing on known quantities $\bar{R}_{n, m}$, defined by equations (1) and (6), the coefficients $\bar{A}_{n, m}$ can be synthesized from (95) for $|m|=1,2, \ldots, N$ and $n=\max \{2,|m|\}, \ldots, N$ by a recurrent procedure. Then from (93) the coefficients $\bar{A}_{1, m}$ are found for $|m|=1$.

\section{CHARACTERISTICS OF THE GRAVITY GRADIENTS}

We consider the disturbing potential $T$ which represents the difference between EGM2008 geopotential model and GRS80 normal field (Moritz, 1980).

The computations are carried out on PC Pentium IV $2.8 \mathrm{GHz}$ with the mantissa of 32 digits. The calculated quantities are expressed in Eötvös units (E).

The associated Legendre functions are calculated on the basis of simple recurrence procedures given by Holmes and Featherstone (2002). The coincidence of the values of the gravity gradients (GG), computed from the new expressions (7)-(11) and from the expressions (4), is established with the precision $10^{-30} \mathrm{E}$.

The recurrent spectral relations derived in Sec. 3 are solved very quickly, taking only 12 seconds for all six derivatives. 
The spectral coefficients of GG are synthesized on the basis of EGM2008 geopotential model which contains the harmonics up to degree and order 2160. From these coefficients the root mean square degree variances $\left(\delta_{n}\right)$ are evaluated and presented in Fig. 1.

Let as note that at the altitude $h=0 \mathrm{~km}$ the convergence of the series for the second order potential derivatives is too slow for a possibility of their implementation. Since the partial sums of the $N$-th degree of the constructed series are equal to the sums of the same degree of the conventional series, as well as the series derived in P2008 (given now in equation (4)), then all three kinds of the series have the same rate of the convergence. The poor convergence at $h=0 \mathrm{~km}$ is explained by the fact that, due to double differentiation, the increase of the general term, proportional to $n^{2}$ (as in equations (5) and (6)), is not compensated by the factor $(a / r)^{n+3}$ since $r=a$.

Taking into account the above comment, we present in Fig. 1 the cases of $h=50,100,250 \mathrm{~km}$ which demonstrate the evolution of the rate of decreasing the degree variances with increasing the altitude. For all GG the degree variances are almost monotonically decreasing and the curves are almost parallel. As was expected, for $T_{z z}$ they have the maximal power and for $T_{x y}$ the minimal one, as compared to the other derivatives. For the latter derivatives the curves are very close to each other.

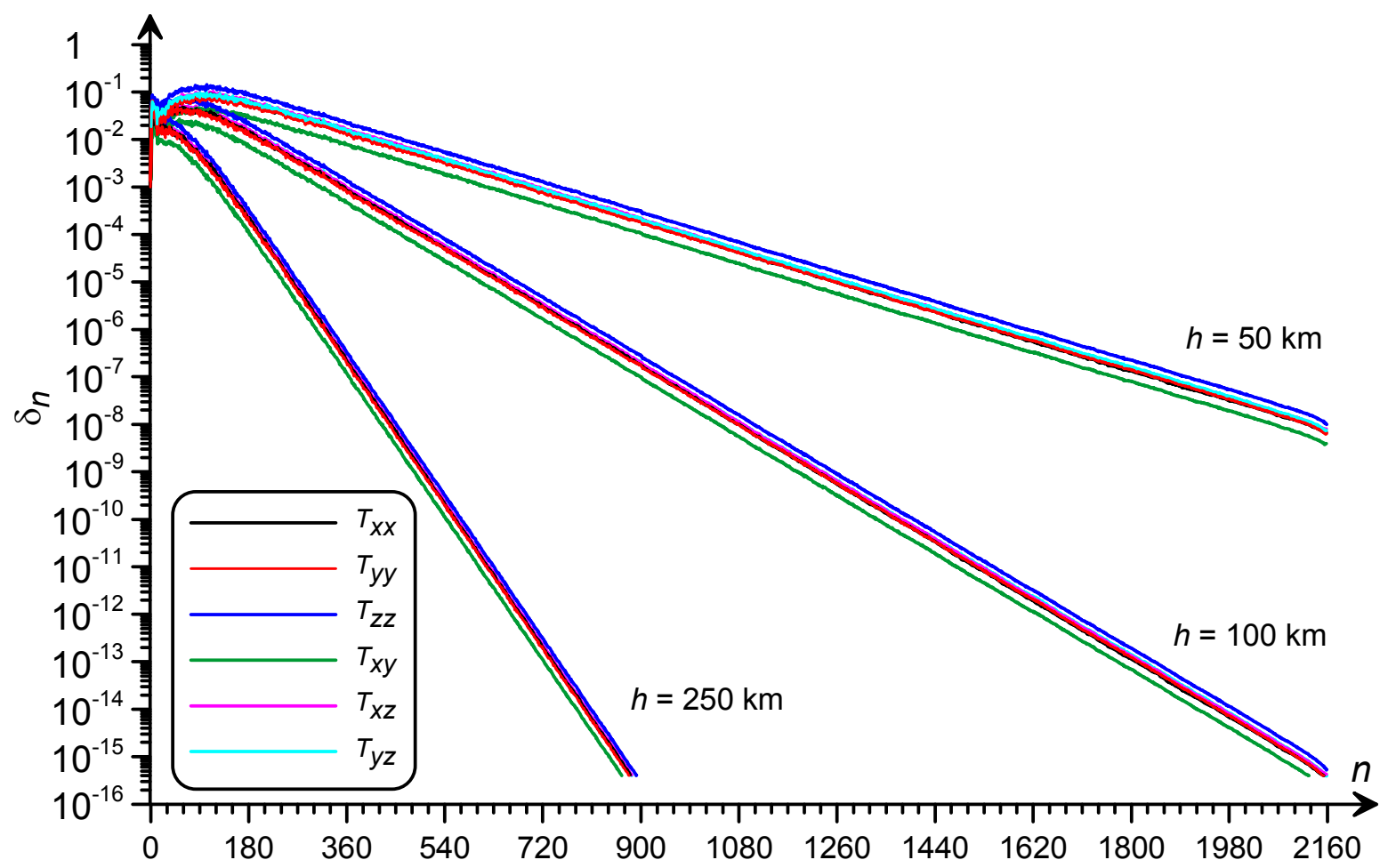

Fig. 1. RMS degree variances $\left(\delta_{n}\right)$ for derivatives $T_{x x}, T_{y y}, T_{z z}, T_{x z}, T_{y z}, T_{x y}$

If one compares the non-orthogonal expansions for GG, presented either by the conventional series or the new ones, given in (4), with the orthogonal series defined in (7)(11), then it can be seen that the latter expressions are much more simple and therefore more appropriate for performing spherical harmonic synthesis and analysis.

We apply expressions (7)-(11) for constructing colored digital maps for Fennoscandia area, which is of interest for geophysicists. In Grafarend and Voosoghi (2003) much attention 
is given to this region, which is "presently undergoing recent crustal uplift as a result of postglacial rebound" (ibid., p. 311).

We construct the maps for the altitude $h=100 \mathrm{~km}$ and not $h=250 \mathrm{~km}$ because the larger is the altitude, the less peculiarities of the gravity gradients are noticeable. These maps visually display the rate of changing the gravity gradients in different directions.

Fig. 2 demonstrates the behavior of all six derivatives. More detailed maps are displayed in Figs. 3 and 4 for $T_{y z}$, because this derivative has the most peculiar behavior as compared with the other ones.

The distribution and the magnitude of the gravity gradient anomalies can be compared with the other known geophysical anomalies. In Fig. 3 the map of isolines for $T_{y z}$ covers the map for the relief data (ETOPO2v2, 2006). This Figure demonstrates the correlation between the gravity gradient anomalies and the relief. Figure 4 , representing the map for $T_{y z}$ covered by a seismic map (CE, 2005), may be of interest for the corresponding specialists in geophysics.

Acknowledgments. The authors are highly thankful to the reviewer for many very valuable comments and recommendations. 


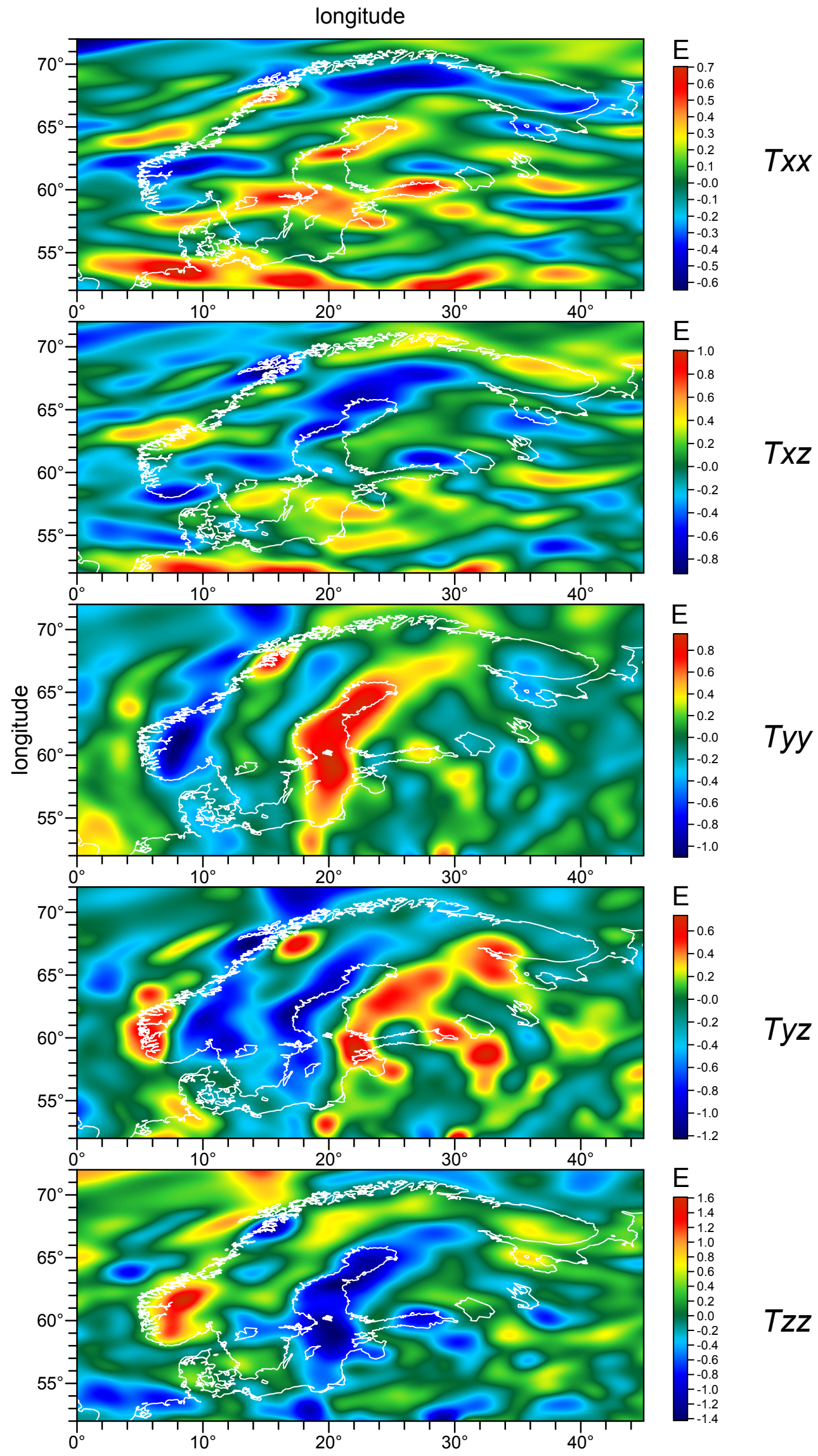

Fig. 2. Maps for derivatives $T_{x x}, T_{y y}, T_{z z}, T_{x z}, T_{y z}, T_{x y}$ for Fennoscandia region 


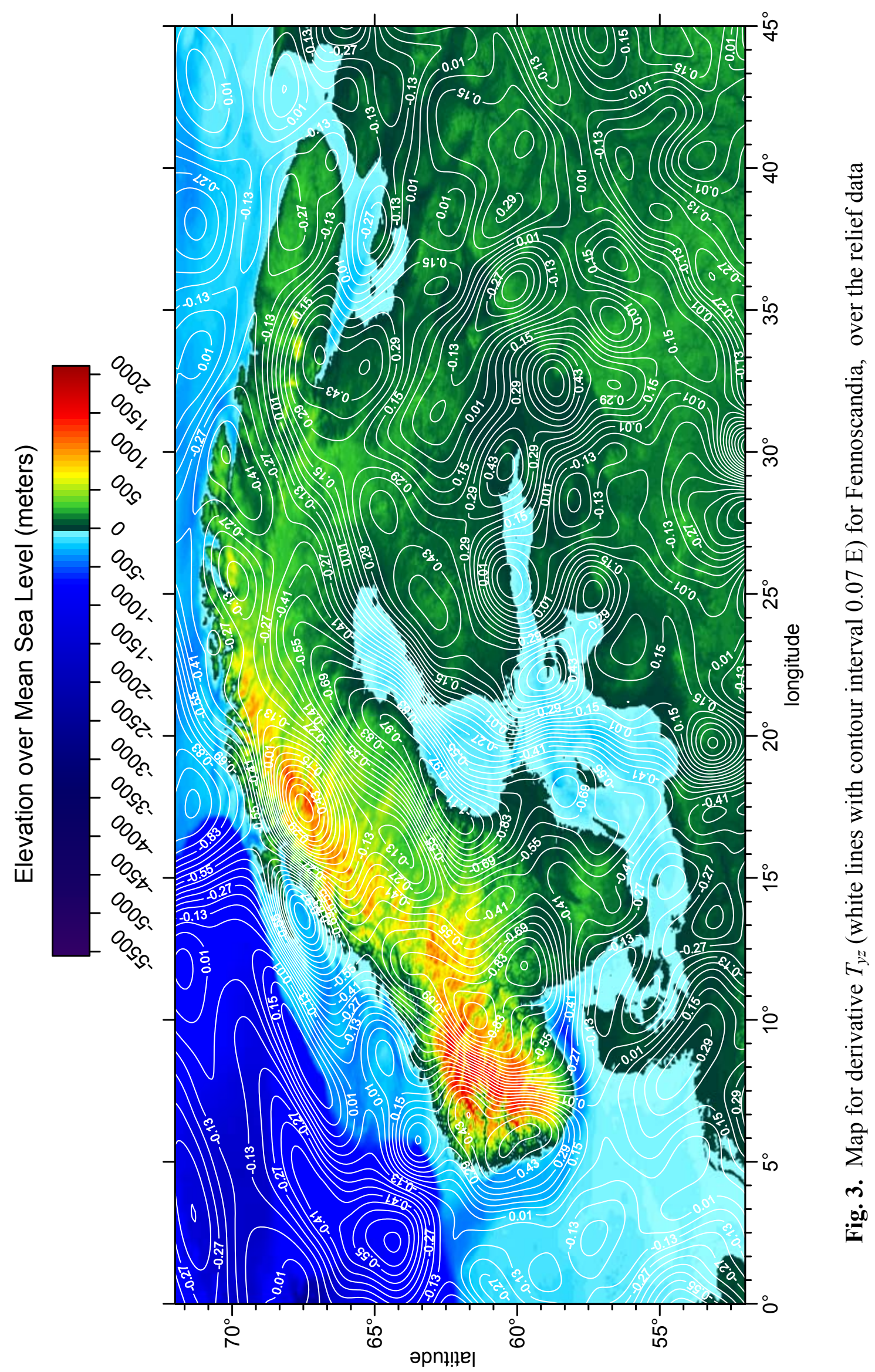




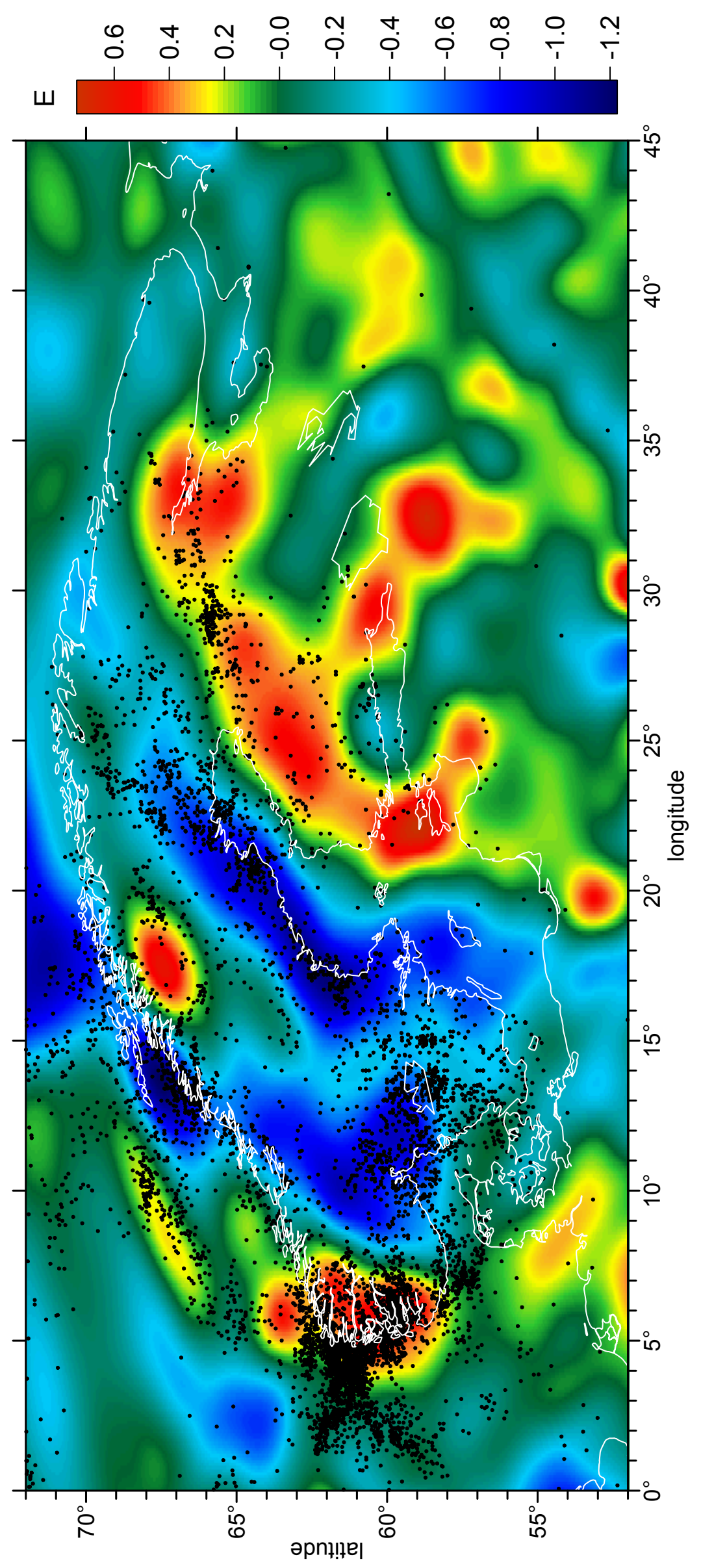

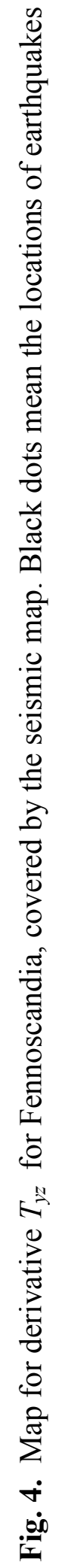


210

APPENDIX: NUMERICAL CONSTANTS

$$
\begin{aligned}
& a_{n, m}=0,|m|=0,1 \\
& a_{n, m}=\frac{\sqrt{1+\delta_{|m|, 2}}}{4} \sqrt{n^{2}-(|m|-1)^{2}} \sqrt{n+|m|} \sqrt{n-|m|+2}, 2 \leq|m| \leq n \\
& b_{n, m}=\frac{(n+|m|+1)(n+|m|+2)}{2(|m|+1)},|m|=0,1 \\
& b_{n, m}=\frac{n^{2}+m^{2}+3 n+2}{2}, 2 \leq|m| \leq n \\
& c_{n, m}=\frac{\sqrt{1+\delta_{|m|, 0}}}{4} \sqrt{n^{2}-(|m|+1)^{2}} \sqrt{n-|m|} \sqrt{n+|m|+2},|m|=0,1 \\
& c_{n, m}=\frac{1}{4} \sqrt{n^{2}-(|m|+1)^{2}} \sqrt{n-|m|} \sqrt{n+|m|+2}, 2 \leq|m| \leq n \\
& n=2,3, \ldots, N \\
& d_{n, m}=0,|m|=0,1 \\
& d_{n, m}=-\frac{m}{4|m|} \sqrt{\frac{2 n+1}{2 n-1}} \sqrt{1+\delta_{|m|, 2}} \sqrt{n^{2}-(|m|-1)^{2}} \\
& \times \sqrt{n+|m|} \sqrt{n+|m|-2}, \quad 2 \leq|m| \leq n \\
& g_{n, 0}=0 \\
& g_{n, m}=\frac{m}{4|m|} \sqrt{\frac{2 n+1}{2 n-1}} \sqrt{n+1} \sqrt{n-1}(n+2),|m|=1 \\
& g_{n, m}=\frac{m}{2} \sqrt{\frac{2 n+1}{2 n-1}} \sqrt{n+|m|} \sqrt{n-|m|}, 2 \leq|m| \leq n \\
& h_{n, 0}=0 \text {, } \\
& h_{n, m}=\frac{m}{4|m|} \sqrt{\frac{2 n+1}{2 n-1}} \sqrt{n-3} \sqrt{n-2} \sqrt{n-1} \sqrt{n+2},|m|=1 \\
& \begin{aligned}
h_{n, m} & =\frac{m}{4|m|} \sqrt{\frac{2 n+1}{2 n-1}} \sqrt{n^{2}-(|m|+1)^{2}} \sqrt{n-|m|} \sqrt{n-|m|-2}, 2 \leq|m| \leq n, \\
n & =2,3, \ldots, N
\end{aligned}
\end{aligned}
$$




$$
\begin{aligned}
& \beta_{n, 0}=0 \\
& \beta_{n, m}=\frac{n+2}{2} \sqrt{1+\delta_{|m|, 1}} \sqrt{n+|m|} \sqrt{n-|m|+1}, 1 \leq|m| \leq n \\
& \gamma_{n, 0}=-(n+2) \sqrt{\frac{n(n+1)}{2}} \\
& \gamma_{n, m}=-\frac{n+2}{2} \sqrt{n-|m|} \sqrt{n+|m|+1}, 1 \leq|m| \leq n, \\
& n=2,3, \ldots, N \\
& \mu_{n, 0}=0 \\
& \mu_{n, m}=-\frac{m}{|m|}\left(\frac{n+2}{2}\right) \sqrt{\frac{2 n+1}{2 n-1}} \sqrt{1+\delta_{|m|, 1}} \sqrt{n+|m|} \sqrt{n+|m|-1}, 1 \leq|m| \leq n \\
& v_{n, 0}=0 \\
& v_{n, m}=-\frac{m}{|m|}\left(\frac{n+2}{2}\right) \sqrt{\frac{2 n+1}{2 n-1}} \sqrt{n-|m|} \sqrt{n-|m|-1}, 1 \leq|m| \leq n, \\
& n=2,3, \ldots, N \\
& \delta_{p, q}= \begin{cases}1, & p=q \\
0, & p \neq q\end{cases} \\
& \bar{g}_{n, m}=\frac{\sqrt{2 n-3} \sqrt{n^{2}-m^{2}} \sqrt{(n-1)^{2}-m^{2}}}{(2 n-1) \sqrt{2 n+1}(n-1) n},|m|=0,1, \ldots, N, \\
& n=\max \{4,|m|+2\}, \ldots, N+2 \\
& \left.\bar{h}_{n, m}=\frac{n+2 m^{2}+1}{(2 n+3)(n+1)},|m|=0,1, \ldots, N, n=\max \{2,|m|\}, \ldots, N\right\} \\
& \left.\tilde{a}_{n, m}=-\frac{(n-|m|+1)(n-|m|+2)}{(2 n+1)(2 n+3)}\right) \\
& \tilde{b}_{n, m}=2 \frac{n^{2}+m^{2}+n-1}{(2 n-1)(2 n+3)} \\
& \tilde{c}_{n, m}=-\frac{(n+|m|)(n+|m|-1)}{(2 n+1)(2 n-1)} \\
& \left.\bar{a}_{n, m}=\frac{\sqrt{n^{2}-m^{2}} \sqrt{(n-1)^{2}-m^{2}}}{(2 n-1) \sqrt{2 n+1)} \sqrt{2 n-3}},|m|=0,1, n=4,5, \ldots, N+2\right) \\
& \bar{b}_{n, m}=2 \frac{n^{2}+m^{2}+n-1}{(2 n-1)(2 n+3)},|m|=0,1, n=2,3, \ldots, N \\
& \bar{c}_{n, m}=\bar{a}_{n+2, m}, \quad|m|=0,1, n=2,3, \ldots, N-2
\end{aligned}
$$




$$
\begin{aligned}
& \left.\begin{array}{l}
\bar{b}_{2,0}^{*}=\frac{12}{35}, \bar{b}_{3,0}^{*}=\frac{20}{63}, \bar{b}_{n, 0}^{*}=\bar{b}_{n, 0}, \quad n=4,5, \ldots, N \\
\bar{b}_{2, m}^{*}=\bar{b}_{2, m}, \bar{b}_{3, m}^{*}=\frac{10}{21}, \bar{b}_{n, m}^{*}=\bar{b}_{n, m}, \quad|m|=1, n=4,5, \ldots, N
\end{array}\right\} \\
& \sigma_{n,|m|}=\frac{1}{\sqrt{1+\delta_{|m|, 2}}} \frac{\sqrt{2 n-3}}{\sqrt{2 n+1}} \sqrt{n+|m|-3} \sqrt{n+|m|-2)} \sqrt{n+|m|-1} \sqrt{n+|m|}, \\
& |m|=2,3, \ldots, N, n=|m|,|m|+1, . ., N+2 \\
& \left.\bar{a}_{n, m}=\frac{\sigma_{n, m}}{(2 n-3)(2 n-1)},|m|=2,3, \ldots, N, n=|m|,|m|+1, \ldots, N+2\right) \\
& \bar{b}_{n, m}=-2 \frac{\sigma_{n+2, m}}{(2 n-1)(2 n+3)} \frac{\sqrt{2 n+5}}{\sqrt{2 n+1}} \frac{\sqrt{n-|m|+1} \sqrt{n-|m|+2}}{\sqrt{n+|m|+1} \sqrt{n+|m|+2}}, \\
& |m|=2,3, \ldots, N, n=|m|,|m|+1, \ldots, N \\
& \bar{c}_{n, m}=\frac{\sigma_{n+4, m}}{(2 n+3)(2 n+5)} \frac{\sqrt{2 n+9}}{\sqrt{2 n+1}} \frac{\sqrt{n-|m|+1} \sqrt{n-|m|+2}}{\sqrt{n+|m|+1} \sqrt{n+|m|+2}} \\
& \times \frac{\sqrt{n-|m|+3} \sqrt{n-|m|+4}}{\sqrt{n+|m|+3} \sqrt{n+|m|+4}} \\
& |m|=2,3, \ldots, N-2, n=|m|,|m|+1, \ldots, N-2 \\
& \hat{a}_{n, m}=\bar{a}_{n, m}-\bar{g}_{n, m}, \quad|m|=0,1, \quad n=4,5, \ldots, N+2 \\
& \left.\hat{b}_{n, m}=\bar{b}_{n, m}^{*}-\bar{h}_{n, m}, \quad|m|=0,1, \quad n=2,3, \ldots, N\right\} \\
& p_{n, m}=\frac{\left(n^{2}-3 n+3\right) \sqrt{n^{2}-m^{2}} \sqrt{(n-1)^{2}-m^{2}}}{(2 n-1) \sqrt{2 n+1} \sqrt{2 n-3} n(n-1)}, \\
& |m|=2,3, \ldots, N, n=|m|+2,|m|+3, \ldots, N+2 \\
& q_{n, m}=-\frac{(n+1)\left(2 n^{2}-1\right)-2 m^{2}(n-2)}{(n+1)(2 n-1)(2 n+3)}, \\
& |m|=2,3, \ldots, N, n=|m|,|m|+1, \ldots, N \\
& r_{n, m}=\frac{\sqrt{(n+1)^{2}-m^{2}} \sqrt{(n+2)^{2}-m^{2}}}{\sqrt{2 n+1} \sqrt{2 n+5}(2 n+3)}, \\
& |m|=2,3, \ldots, N-2, n=|m|,|m|+1, \ldots, N-2\}
\end{aligned}
$$




$$
\begin{aligned}
& \bar{\xi}_{2, m}=0,|m|=1 \\
& \bar{\xi}_{n, m}=\frac{m(n-2) \sqrt{n^{2}-m^{2}}}{n(n+1) \sqrt{4 n^{2}-1}} \\
& |m|=1,2, \ldots, N, n=\max \{3,|m|+1\}, \ldots, N+1\} \\
& \bar{\eta}_{n, m}=\frac{m \sqrt{(n+1)^{2}-m^{2}}}{(n+2) \sqrt{2 n+1} \sqrt{2 n+3}} \\
& |m|=1,2, \ldots, N-1, n=|m|,|m|+1, \ldots, N-1 \\
& \bar{b}_{1, m}=\frac{4}{5}, \bar{c}_{1, m}=\frac{2 \sqrt{2}}{5 \sqrt{7}}, \bar{a}_{3, m}=\frac{2 \sqrt{2}}{5 \sqrt{7}},|m|=1 . \\
& \alpha_{n, m}=\frac{1}{\sqrt{1+\delta_{|m|, 1}}} \frac{\sqrt{2 n-1}}{\sqrt{2 n+1}} \sqrt{n+|m|-1} \sqrt{n+|m|}, \\
& |m|=1,2, \ldots N, n=|m|,|m|+1, \ldots, N+1 \\
& \bar{\beta}_{2, m}=0,|m|=1 \\
& \left.\bar{\beta}_{n, m}=\frac{(n-1) \sqrt{n^{2}-m^{2}}}{n \sqrt{4 n^{2}-1}},|m|=1,2, \ldots, N, n=\max \{3,|m|+1\}, \ldots, N+1\right\} \\
& \bar{\gamma}_{n, m}=\frac{\sqrt{(n+1)^{2}-m^{2}}}{\sqrt{(2 n+1)} \sqrt{(2 n+3)}},|m|=1,2, \ldots, N-1, n=|m|, \ldots, N-1 \\
& \bar{\mu}_{n, m}=\sqrt{\frac{(n+|m|)(n+|m|-1)}{\left(1+\delta_{|m|, 1}\right)\left(4 n^{2}-1\right)}}, \\
& |m|=1,2, \ldots, N, n=|m|,|m|+1, \ldots, N+1 \\
& \bar{v}_{n, m}=\sqrt{\frac{(n-|m|+1)(n-|m|+2)}{\left(1+\delta_{|m|, 1}\right)(2 n+1)(2 n+3)}}, \\
& |m|=1,2, \ldots, N-1, n=|m|,|m|+1, \ldots, N-1 \mid \\
& \hat{\mu}_{n, m}=\bar{\mu}_{n, m} \frac{n+1}{m}, \\
& |m|=1,2, \ldots, N, n=\max \{2,|m|\}, \ldots, N \\
& \hat{v}_{n, m}=\bar{v}_{n, m} \frac{n+1}{m} \text {, } \\
& |m|=1,2, \ldots, N-2, n=\max \{2,|m|\}, \ldots, N-2\}
\end{aligned}
$$




\section{REFERENCES}

CE (2005) Catalog of earthquakes in Northern Europe 1375-2005. The Institute of Seismology of the University of Helsinki. http://www.seismo.helsinki.fi/bulletin /list/catalog/Scandia_clean.html

Claessens SJ New relations among associated Legendre functions and spherical harmonics. Journal of Geodesy, vol 79: 398-406.

ETOPO2v2 (2006) 2-minute Gridded Global Data. U.S. Department of Commerce, National Oceanic and Atmospheric Administration, National Geophysical Data Center. http://www.ngdc.noaa.gov/mgg/fliers/06mgg01.html

Grafarend E. W., Voosoghi B. (2003) Intrinsic deformation analysis of the Earth's surface based on displacement fields derived from the space geodetic measurements. Case studies: present-day deformation patterns of Europe and of the Mediterranean area (ITRF data sets). Journal of Geodesy, Vol. 77, No. 5-6, pp. 303-326.

Heiskanen W. A., Moritz H. (1979) Physical Geodesy. Institute of Physical Geodesy, Technical University, Graz, Austria.

Holmes S. A., Featherstone W. E. (2002) A unified approach to the Clenshaw summation and the recursive computation of very high degree and order normalized associated Legendre functions. Journal of Geodesy, Vol. 76, No. 5, pp. 279-299.

Ilk KH (1983) Ein Beitrag zur Dynamik ausgedehnter Körper - Gravitationswechselwirkung. Deutsche Geodätische Kommission, Reihe C, Heft Nr. 288, München.

Moritz H (1980) Geodetic Reference System 1980. Bull Géod 54 (4): 395-405

Pavlis NK, Holmes SA, Kenyon SC, Factor JK (2008) An Earth Gravitational Model to Degree 2160: EGM2008, presented at the 2008 General Assembly of the European Geosciences Union, Vienna, Austria, April 13-18

Petrovskaya M. S., Vershkov A. N. (2006) Non-singular expressions of the gravity gradients in the local north-oriented and orbital reference frames. Journal of Geodesy, Vol. 80, No. 3, pp. 117-127.

Petrovskaya M.S., Vershkov A. N. (2008) Development of the local north-oriented second order derivatives of the Earth potential in orthogonal series of modified spherical harmonics. Journal of Geodesy, Vol. 82, No. 12, pp. 929-944, DOI: 10.1007/s00190008-0223-z.

Received: 2008-04-22,

Reviewed: 2009-01-08, by J. Bouman,

Accepted: 2009-01-21. 